DOI : 10.3901/JME.2021.09.233

\title{
超声振动辅助磨料流抛光技术研究综述
}

\author{
李 华 ${ }^{1,2}$ 任 坤 ${ }^{1,2}$ 殷 振 ${ }^{1,2}$ 戴晨伟 ${ }^{1,2}$ \\ 王中旺 $^{1}$ 魏 力 $^{1}$ 韦 冬 ${ }^{1}$
}

(1. 苏州科技大学机械工程学院 苏州 215009;

2. 苏州科技大学苏州市精密与高效加工技术重点实验室 苏州 215009)

摘要: 抛光加工是实现高性能零件最终表面质量要求的重要的方法。超声振动辅助磨料流抛光是磨料流抛光与超声振动相结 合的复合抛光技术，在光学玻璃、蓝宝石、单晶硅、陶瓷等硬脆性材料抛光和金属材料零件复杂表面的抛光加工中具有独特 的优势, 可以有效地提高表面质量和抛光效率。对近年来的技术发展进行了总结, 对抛光加工基本原理研究、材料去除微观 机理研究、抛光工艺方法分类及应用等方面进行了系统的论述和分析。指出了这一技术当前的研究热点和需要解决的关键问 题, 对今后的研究、应用和发展给出了建议和展望。

关键词: 超声振动; 磨料流抛光; 去除机理

中图分类号: TG663

\section{Review of Ultrasonic Vibration-assisted Abrasive Flow Polishing Technology}

\author{
LI Hua ${ }^{1,2}$ REN Kun ${ }^{1,2}$ YIN Zhen ${ }^{1,2}$ DAI Chenwei ${ }^{1,2}$ \\ WANG Zhongwang ${ }^{1}$ WEI Li ${ }^{1}$ WEI Dong ${ }^{1}$
}

(1. School of Mechanical Engineering, Suzhou University of Science and Technology, Suzhou 215009;

2. Suzhou Key Laboratory of Precision and Efficient Machining Technology,

Suzhou University of Science and Technology, Suzhou 215009)

\begin{abstract}
Polishing is an important method to achieve the final surface quality requirements of high-performance parts. Ultrasonic vibration assisted abrasive flow polishing (UVAFP) is a compound polishing technology combining abrasive flow polishing technology with ultrasonic vibration technology. It is of unique advantages in the polishing of complex surfaces of hard brittle materials such as optical glass, sapphire, monocrystalline silicon, ceramics and metal parts, which can effectively improve the surface quality and polishing efficiency. The development of this technology in recent years is summarized, and the basic principle of polishing, the micro mechanism of material removal, the classification of polishing process and its application are discussed and analyzed systematically. The current research hotspots and key problems of this technology were pointed out, and suggestions and prospects for the future research, application and development of this technology are proposed.
\end{abstract}

Key words: ultrasonic vibration; abrasive flow polishing; removal mechanism

\section{0 前言}

抛光加工是实现零件最终表面质量要求的重要 的方法, 是精密与超精密加工的重要技术手段之 - ${ }^{[1-2]}$ 。尤其是对于采用石英玻璃、单晶硅、蓝宝石、

* 国家自然科学基金(51905363)和江苏省自然科学基金(BK20190940)资 助项目。20200515 收到初稿, 20210210 收到修改稿
精密陶瓷、超硬材料等硬脆性难加工材料的精密元 件和高精度滚动体等零件, 抛光加工几乎是达到超 高表面质量的唯一途径 ${ }^{[3-7]}$ 。抛光加工技术是以抛光 磨具、抛光介质等为工具, 在机械、化学、电场、 磁场等能量的作用下, 从被加工工件表面去除微量 的材料, 降低工件表面粗糙度、提高表面质量的工 艺方法。根据所用的抛光工具的不同, 可以分为固 结磨具抛光、柔性磨具抛光、磨料流体抛光等; 根 
据起作用的主要能量形式不同, 可以分为机械式抛 光、流体抛光、多能场复合抛光等方法 ${ }^{[8-10]}$ 。其中 机械式抛光和流体抛光是基础方法。随着新材料的 广泛应用和对复杂形状零件表面质量要求的不断提 高, 电化学抛光、磁 (电) 流变抛光、激光抛光等新 型的精密高效复合抛光技术也被提出 ${ }^{[11-18]}$ 。

磨料流抛光(Abrasive flow polishing, AFP) 是以 磨料和液体混合而成的流体为介质, 在外部能量场 的推动下作用于被加工工件的表面, 实现加工表面 的微量材料去除, 从而降低工件表面粗糙度等微观 缺陷的抛光技术 ${ }^{[19]}$ 。AFP 属于非接触或半接触式抛 光法, 在对球面、非球面等曲面形状工件表面、空 间结构表面、内表面以及其他复杂形状表面的精密 抛光加工中具有显著的优势。磨料流抛光加工可以 分为磨料流流体抛光、磨料流射流抛光、柔性垫流 体抛光三大类 ${ }^{[20-23]}$ 。其中, 前两类是依靠磨料流体 介质完成抛光的非接触式抛光方法; 第三类方法是 利用柔性垫加上磨料流对工件表面进行半接触式拋 光的方法, 这是目前单晶硅基片、光学镜片、蓝宝 石等硬脆性材料表面主要的超精密抛光方法。在柔 性垫流体抛光中, 抛光垫是由具有大量微孔隙的柔 性材料制成, 其与工件表面之间存在局部凸峰处实 体接触和孔隙处不接触两种状态, 加入磨料流体后, 两者处于半接触的边界润滑摩擦状态 ${ }^{[23]}$ 。抛光垫与 工件表面之间相对转动, 带动磨料实现抛光加工。 其抛光过程包涵了接触部位磨粒对工件表面的挤压 与微切削作用和非接触部位流体抛光作用。

超声振动辅助磨料流抛光技术 (Ultrasonic assistant abrasive flow polishing UVAFP) 是把超声振 动与磨料流抛光相结合形成的复合抛光加工技 术 ${ }^{[24-25]}$ 。这种抛光技术尤其适用于硬脆性难加工材 料表面、复杂形状表面的高效精密抛光, 拓展了磨 料流抛光技术的工艺范围, 提高了抛光加工效率和 抛光表面质量。国内外学者对 UVAFP 进行了广泛 深入的研究, 把超声振动技术与各种磨料流抛光技 术的结合, 提出了多种超声辅助磨料流抛光方法, 在多种材料的精密抛光取得了优良的效果。本文对 近年来国内外在 UVAFP 研究领域取得的进展进行 总结与分析, 并对今后的发展进行展望。

\section{UVAFP 工作原理}

UVAFP 技术是通过超声振动系统把超声振动 作用于磨料流, 由超声振动能量与流体动能相结合 完成抛光加工。如图 1 所示, 在超声电源的电信号
驱动下, 压电陶瓷换能器带动变幅杆与工具产生超 声频振动。工具端面把超声振动传入磨料流。在超 声振动作用下, 增强了磨料流对被加工工件表面的 冲击作用 ${ }^{[26]}$, 实现了被加工表面的高效抛光加工。 在应用研究中, 有工具超声振动和工件超声振动两 种形式。其共同的效果都是对磨料流施加超声振动, 增强其抛光能力。

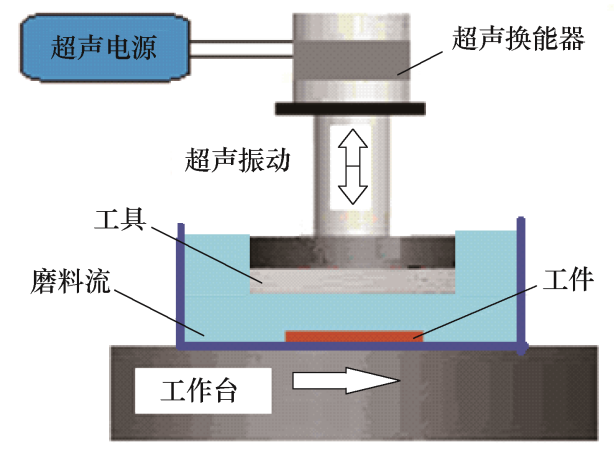

图 1 超声辅助磨料流抛光示意图

研究表明: 在超声振动的作用下, 磨料流对工 件表面的抛光作用有三种方式 ${ }^{[26]}$ 。

一是磨料流的流体动力带动磨粒对工件表面的 冲蚀作用。液体中的超声振动声场使液体产生紊流, 改变了流体的速度和方向, 增加了流体的动能, 流 体运动带动磨粒冲蚀工件表面从而实现微去除。

二是超声振动直接驱动磨粒对工件表面冲击作 用。当工具与工件表面距离较近时, 超声振动工具 的振动辐射面直接驱动液体中的磨粒沿振动方向高 速运动, 对工件表面产生冲击, 使工件表面产生微 塑性变形和微破损去除。

三是超声空化射流对工件表面的冲击作用。在 超声振动作用下, 液体中产生空化现象, 空化泡溃 破产生的高压射流推动液体和磨粒冲击工件表面, 产生微去除作用。

UVAFP 过程是上述三种作用方式综合作用的 结果, 但在不同的方法中, 起主要作用的方式也不 同。在 UVAFP 加工中, 由于抛光工具与工件不接 触或仅有局部的弹性接触, 对工件的作用力和热影 响小, 适用于硬脆材料表面、复杂曲面以及薄壁、 薄片等低刚度工件表面的抛光。把 UVAFP 与射流、 挤压流等流体的动能相结合或与电化学等方法结 合, 可以等进一步增强磨粒对工件表面的作用效果, 提高抛光效率。

\section{UVAFP 加工方法的研究}

超声振动与传统磨料流抛光相结合, 产生了多 
种形式的 UVAFP 加工方法。按超声振动施加对象 不同, 可分为工具超声振动、工件超声振动、流场 超声振动等方式; 按超声振动类型不同可分为单向 超声振动、椭圆超声振动、两维超声振动、阵列式 超声振动、聚焦超声振动等; 按与超声振动复合的 传统抛光方法不同可分为超声振动磨料流冲击抛 光、超声振动磨料射流抛光、超声振动挤压磨料流 抛光、超声振动柔性垫流体抛光等。本文根据超声 振动能量在抛光过程中所起作用的不同以及与超声 振动相结合的抛光方法不同进行论述。

在 UVAFP 加工中, 根据抛光方法的不同, 磨 料流在抛光过程中的可以分为两种状态: 一种是处 于低速流动或准静态的状态, 这时磨料流本身不具 有抛光加工的动能, 需要在超声振动的驱动下才能 完成抛光, 称为超声振动磨料流抛光, 包括超声振 动磨料流冲击抛光、超声振动磨料流场抛光等; 另 一种是处于高速或高压流动状态, 这时磨料流具有 抛光的动能, 与超声振动复合后可以实现更高效的 抛光, 称为超声振动磨料流复合抛光, 包括超声振 动磨料射流抛光、超声振动磨料流挤压抛光等。

\section{1 超声振动磨料流冲击抛光}

在超声振动磨料流冲击抛光时, 工具与工件表 面之间被磨料流隔开, 超声振动系统带动抛光工具 (或工件)产生超声振动(图 1)。在超声振动作用下, 磨料流中的磨粒冲击工件表面, 去除微量材料, 实 现表面抛光。这种方法具有结构简单, 使用方便等 优点。

(1) 纵向超声振动磨料流抛光

超声振动磨料流冲击抛光多采用工具纵向振动 方式。如 RALCHENKO 等 ${ }^{[27]}$ 采用 $22 \mathrm{kHz}$ 纵向超声 振动工具系统和水基金刚石微粉磨料流, 对金刚石 膜表面进行了抛光加工(图 2), 金刚石膜的表面粗䊁 度在 $5 \mathrm{~min}$ 时间内从 $R a 5 \mu \mathrm{m}$ 减小到 $0.5 \mu \mathrm{m}$ 。加工 效率比传统的抛光方法高两个数量级, 比文献报道 的热机械抛光高出几倍。YU 等 ${ }^{[29-32]}$ 把工具超声振 动与超声雾化后的金刚石抛光液相结合(图 3)。对单 晶硅等材料进行了 UVAFP 加工, 经过 $1 \mathrm{~h}$ 的抛光, 工件的表面粗粮度可由 $100 \mathrm{~nm}$ 左右减小到 $20 \mathrm{~nm}$ 。 超声雾化增强了抛光液在抛光区的渗透性, 减少了

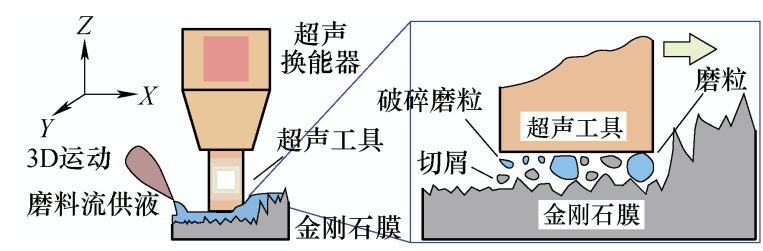

图 2 工具超声振动磨料流抛光
使用量。若工具头施加一定速度的旋转, 对提高超 声振动抛光效率和抛光表面质量具有更显著的作 用。汪帮富等 ${ }^{[36]}$ 采用纵向超声振动系统轴向供液, 把 $\mathrm{SiC}$ 微磨料抛光液雾化后对工件表面进行直接 喷射抛光, 取得了明显的效果, 显著地减少磨料抛 光液的使用量。万宏强等 ${ }^{[35]}$ 在工具纵向超声振动的 基础上, 对工件施加低频振动, 形成高低频复合超 声振动抛光方法。通过对铸铁工件表面的抛光试验, 采用超声复合振动抛光后的工件表面粗䊁度降低了 $14.3 \%$ ，工件表面材料去除量提高了 $24.5 \%$ 。

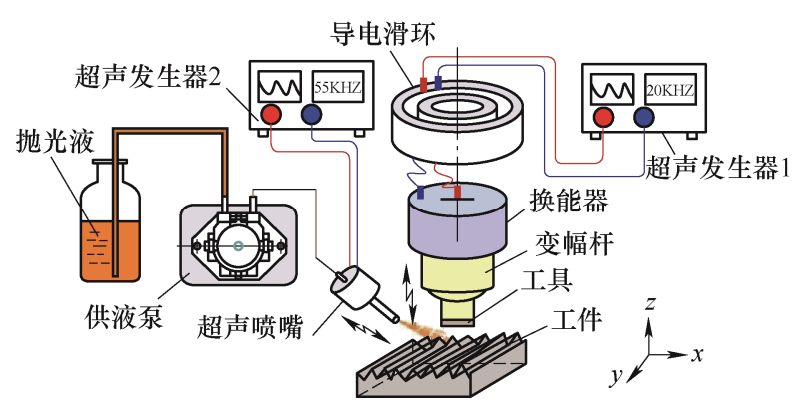

图 3 工具超声振动喷雾抛光

计时鸣等 ${ }^{[33-34]}$ 根据被加工工件的结构化表面形 状(图 4), 设计相应形状的超声振动工具端面, 与被 加工表面共同形成磨料流的约束流道, 工具端面的 超声振动使流道里循环流动的磨料流受到超声振动 激振, 增强湍流强度并产生超声空化, 实现对被加 工表面的抛光, 可以提高结构化曲面的抛光效率和 表面质量, 实现对成形表面的抛光加工。

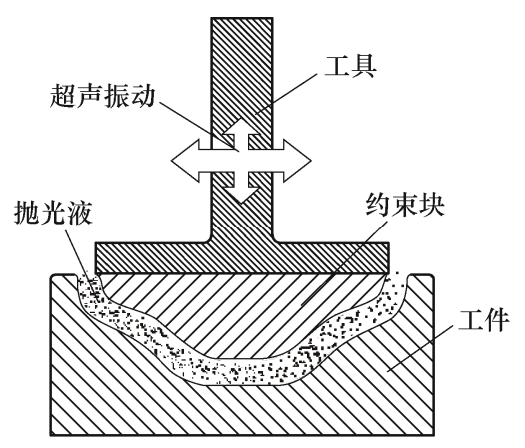

图 4 超声振动型面约束磨料流抛光

为充分利用超声振动的能量, 这些方法需要减小 抛光工具与工件表面之间距离。使工具的超声振动可 以通过很薄的抛光液层作用在工件表面。同时, 工具 头还要满足超声振动系统谐振要求, 其工作端面的大 小和形状都受限制。因而主要用于平面的抛光。

除了工具超声振动, 也有把工件固定在变幅杆 前端, 通过工件的超声振动实现磨料流抛光的研究。 其基本原理与工具振动的方式相同。由于受超声振 动系统振动谐振条件的限制, 这种方式不能适应工 
件结构及大小的变化, 应用中工件的安装也不方便。

近年来有学者应用圆盘弯曲振动或椭圆振动方 式构成工具振动系统。舒晨、李新和等 ${ }^{[28]}$ 利用压电 纵振动换能器与弯曲振动圆盘构成纵弯转换超声振 动抛光工具系统, 采用 $\mathrm{SiC}$ 微粉磨料抛光液, 利用 圆盘端面的超声弯曲振动对石英光学玻璃进行抛 光, 得到粗䊁度值为 $R a 3 \mathrm{~nm}$ 的表面。这是一种值 得探索的抛光方法。

(2) 聚焦超声振动磨料流抛光

BEAUCAMP 等 ${ }^{[38-39]}$ 提出了应用纵向振动超声 换能器和声透镜聚焦对磨料流施加超声振动, 利用 聚焦超声对磨料流的超声空化效应实现高效抛光的 方法, 如图 5 所示。在聚焦超声的作用下, 透镜前 方雉体内的磨料流产生超声振动, 并在出口处聚焦 形成高强度超声振动, 使磨料流产生强烈的空化。 在空化射流的推动下, 磨料冲蚀被加工表面, 实现 抛光加工。采用 $26 \mathrm{kHz}$ 的超声振动系统和 $\mathrm{Al}_{2} \mathrm{O}_{3}$ 水 基磨料流抛光 $\mathrm{BK} 7$ 玻璃和无电镍, 在同样的加工条 件和加工表面质量下, 超声振动抛光的加工效率是 普通抛光的 380\%(图 6)。这一方法实现了超声振动 能量的高效利用, 可以实现对工件表面指定位置的 精确去除, 具有明显的优势, 是一种有应用前景的 新型超声振动磨料流抛光方法。

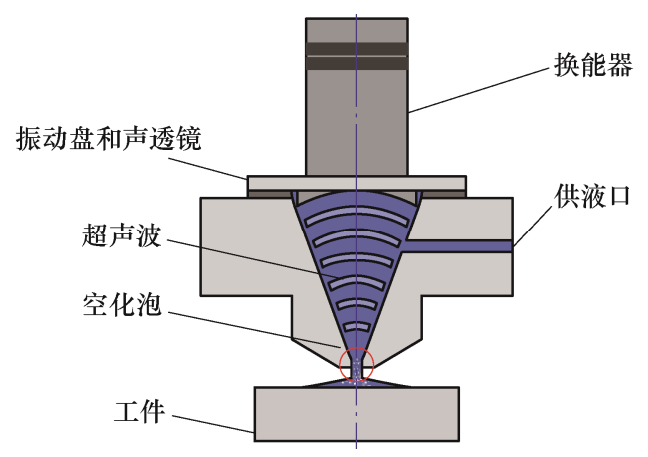

图 5 聚焦超声磨料流抛光

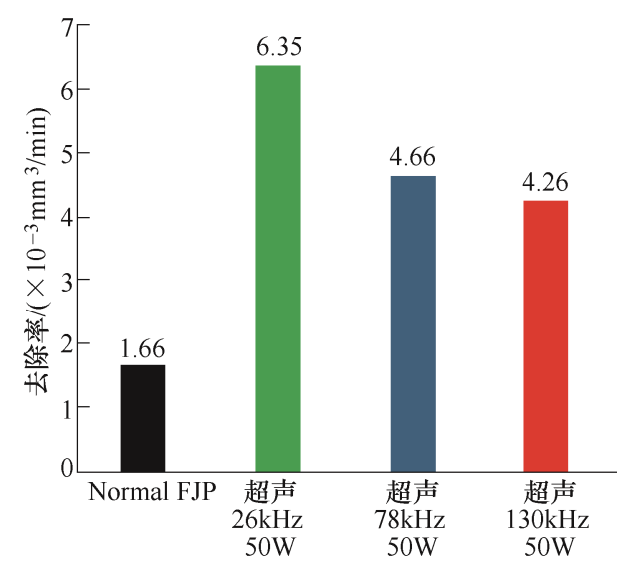

(a) 无电镍

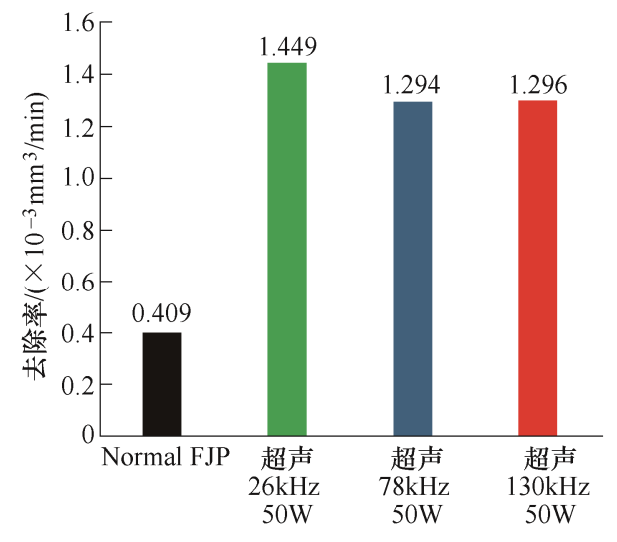

(b) BK7玻璃

图 6 聚焦超声磨料流抛光去除率

\section{2 超声振动磨料流场抛光}

超声振动磨料流场抛光是把工件浸入在磨料 流中的特定位置, 利用超声振动换能器对容器中 的磨料流施加超声振动, 在液体中形成具有一定 强度和分布规律的速度流场, 流体运动带动磨粒 对工件表面产生冲击作用与流场中产生的超声空 化作用相结合完成抛光加工。抛光时工件的低速 回转, 完成整个表面的抛光。受流场强度的限制, 这种方法的加工效率不高, 主要适用于超光滑表 面的微量抛光。

李道朋等 ${ }^{[40-41]}$ 进行了多维超声激励磨料流流 场抛光方法研究。建立了超声振动试验系统 (图 7), 利用多组超声振动振子形成的阵列驱动磨 料流, 使之动产生的复杂的湍流流场, 带动磨粒 冲蚀工件表面。作者研究了振子阵列的分布方式 对抛光液中超声声场的影响。试验表明: 这种抛 光方法能实现超光滑表面抛光, 抛光玻璃表面粗 楉度可以达到纳米级, 抛光不锈钢钢板表面粗糙 度减小率达到 $33 \%$ 。

QI 等 ${ }^{[42]}$ 研究了基于超声悬浮的磨料流场抛 光方法。利用换能器和反射体在磨料流中产生 超声振动驻波声场, 在驻波超声场的作用下, 节点处具有较大的声场辐射力, 推动磨粒沿着 声压线的方向快速运动。将工件置于声压等势 线处, 工件表面就会受到呈现一定方向的磨粒 冲击载荷作用, 置于非等势线位置, 将会受到 湍流的冲击作用(图 8)。同时液体中的超声空化 也使磨粒产生冲击作用，增强材料去除能力。 利用声悬浮装置对硅片和铇钢进抛光试验, 铇 钢试表面粗糙度从抛光前的 $R a 45.99 \mathrm{~nm}$ 降低到 $R a 8.99 \mathrm{~nm}$ 。 


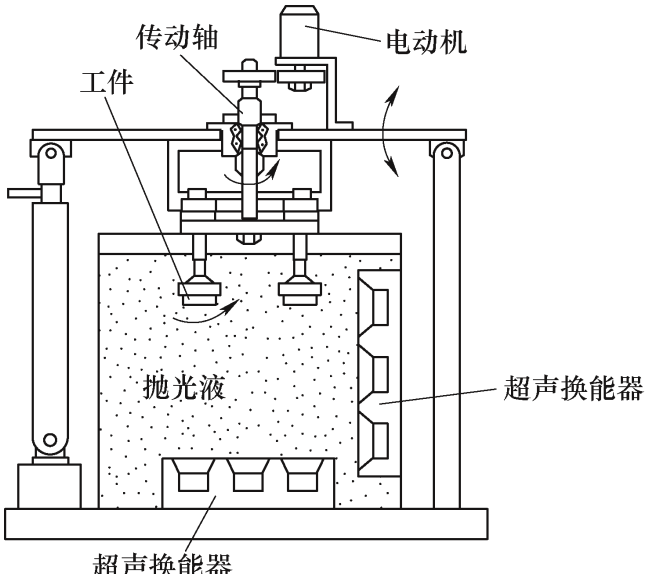

超声换能器

(a) 两维阵列声场

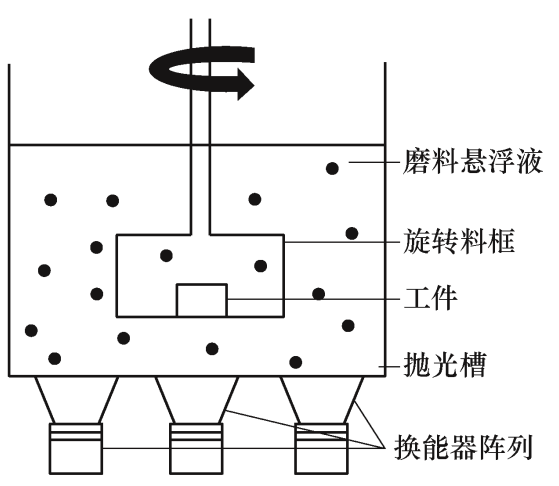

(b) 一维阵列声场

图 7 超声振动磨料流流场抛光

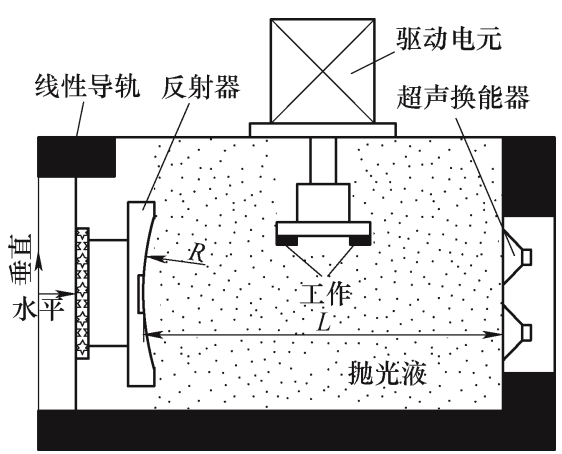

图 8 超声悬浮磨料流抛光

何就等 ${ }^{[37]}$ 针对硅片的抛光, 提出了基于兆赫 级压电振子驱动抛光液喷射抛光方法。固定在旋 转圆盘下端面的被抛光硅片随旋转圆盘做低速 转动 (图 9)。超声振子产生的兆赫级超声振动 $(1.7 \mathrm{MHz})$ 驱动抛光液向上产生涌泉流动, 带动磨 粒冲击旋转的硅片表面, 从而达到抛光的目的。 试验表明, 该方法对硅片的化学机械精密抛光具 有明显效果。在合适的液面高度时, 振子使抛光 液产生很强的涌泉喷射, 可以在距工件表面较远 距离实现抛光效果。经 $60 \mathrm{~min}$ 抛光, 硅片表面粗 糙度最低可达 Ra2.1 nm。

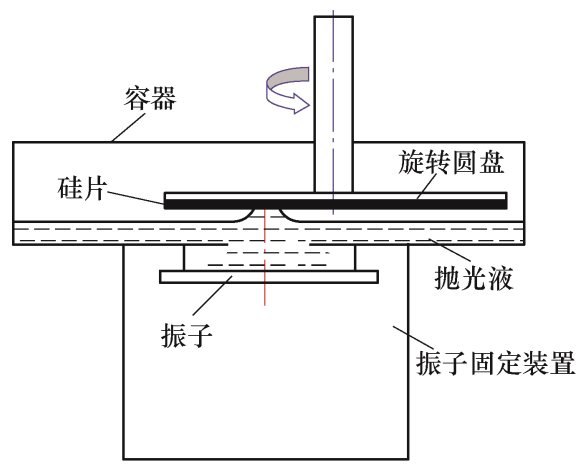

图 9 兆赫超声涌泉喷射抛光

\section{3 超声振动挤压磨料流抛光}

传统的挤压磨料流抛光方法利用压力磨料流对 工件表面循环或往复冲刷, 实现对工件表面抛光 ${ }^{[17]}$ 。 在此基础上, 对被抛光工件施加与磨料流流动方向垂 直的超声声振动, 可以改变磨料流与工件表面的作用 状态, 增加磨粒对工件表面的冲击强度, 形成超声振 动与流体动力复合的抛光方法。

SHARMA 等 ${ }^{[43-45]}$ 把超声振动辅助磨料流抛光 应用于内圆表面、圆雉齿轮齿面抛光(图 10)。通过 液压缸推动磨料流沿工件的轴向往复流动, 同时采 用压电超声振动系统推动工件沿横向(径向)作超声 振动。超声振动改变了工件的有效横截面。提高了 磨粒冲击工件表面的动能, 使抛光效率和表面质量 明显好于普通抛光。轮齿表面粗粘度纹理也得到改 善。应用 250 目 $(58 \mu \mathrm{m}) \mathrm{SiC}$ 磨料流抛光 45 钢内孔,

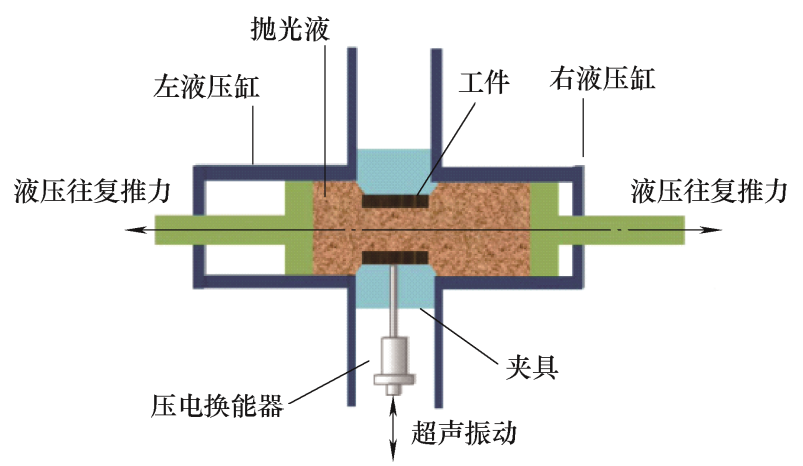

(a) 内孔抛光

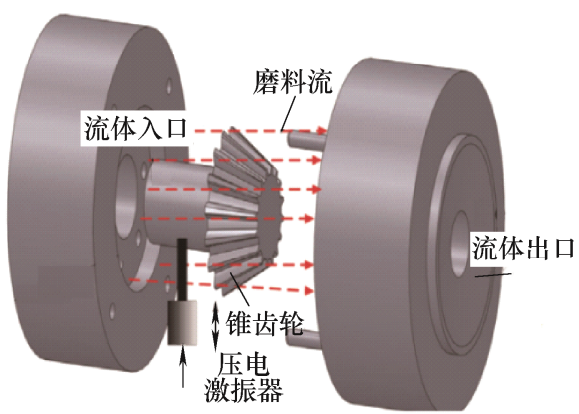

(b) 齿面抛光

图 10 超声振动磨料流复合抛光 
经过 7 9 min 的加工, 内孔表面粗粘度由初始的 $1.98 \mu \mathrm{m}$ 降低到 $0.89 \mu \mathrm{m}$, 表面粗粘度降低率是普通 挤压磨料流抛光的 2.5 倍。应用 220 目 $(68 \mu \mathrm{m}) \mathrm{SiC}$ 磨料流抛光 EN8 钢锥齿轮齿面, 经过 $10 \mathrm{~min}$ 抛光, 齿面粗粘度降低率可达到 $70 \%$ 以上, 是普通挤压磨 料流抛光的 1.5 倍。这种方法受磨料流挤压原理的 限制, 主要适用于金属材料内孔表面的抛光, 且系 统结构较为复杂。

\section{4 超声振动磨料射流抛光}

磨料射流抛光技术是利用高压射流为动力的高 效精密抛光加工技术 ${ }^{[46-48]}$ 。当磨粒的流以一定角度 冲击工件时, 对工件表面产生垂直和水平两个方向 的作用, 垂直方向上作用力对工件表面产生撞击和 挤压作用, 水平方向作用力对工件表面的粗粘凸峰 进行剪切作用, 两方面的综合实现表面抛光。在此 基础上, 通过给喷嘴或工件施加超声振动, 形成了 超声振动磨料射流抛光技术。增加超声振动后, 抛 光工作由磨料射流动能与超声振动共同完成, 综合 了磨料射流和超声振动的优势, 是近年来研究的热 点之一。

吕哲等 ${ }^{[49-51]}$ 分别进行了喷嘴超声振动、工件法 向超声振动、工件切向超声振动和工件扭转超声振 动情况下陶瓷和玻璃超声振动磨料射流抛光的研究 (图 11)。研究表明: 采用工件超声振动方式(包括切 向和法向两种振动方式), 可以增强对工件材料的塑 性剪切去除能力, 降低抛光表面粗糙度, 改善由于 磨粒冲蚀而产生的纹理, 使抛光后表面呈现压应力。 对氮化铝基片的抛光试验, 抛光效率明显提高; 采 用喷嘴超声振动时, 磨料射流叠加了超声振动的动 能, 使射流脉动频率和脉动幅度明显增大, 明显提 高磨料射流冲蚀能力。由于工件振动方式受工件的 形状结构和大小影响, 从应用推广的角度出发, 应 该加强对喷头振动方式的基础和应用研究。

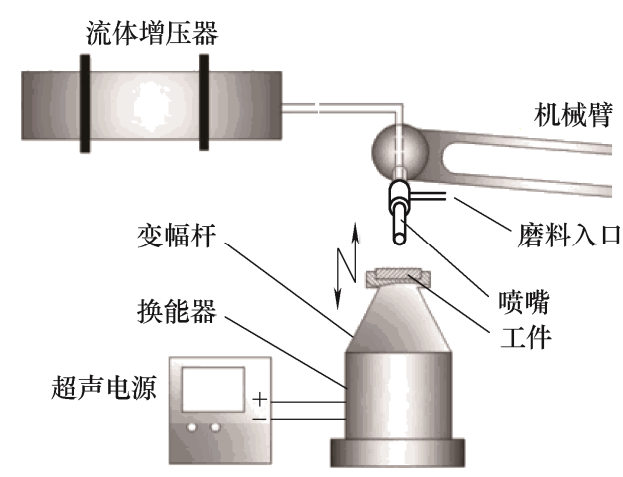

图 11 工件超声振动射流复合抛光

$4 \mathrm{H}-\mathrm{SiC}$ 具有极高的硬度和脆性, 其硬度等级仅 次于金刚石和 CBN, 难以实现高效抛光。NGUYEN
等 ${ }^{[48]}$ 通过对单晶 $4 \mathrm{H}-\mathrm{SiC}$ 薄膜施加垂直于表面的超 声振动(频率 $20 \mathrm{kHz}$ 、振幅 $10 \mu \mathrm{m}$ ), 使用 $\mathrm{SiC}$ 磨料 进行了超声振动磨料射流抛光试验(喷嘴直径 $125 \mu \mathrm{m}$, 射流速度 $120 \mathrm{~m} / \mathrm{s}$ )。在超声振动辅助下, 去除表面的深度比相同条件下的普通磨料射流加工 大 18 倍(图 12), 并且加工表面粗粘度也得到了显著 改善。

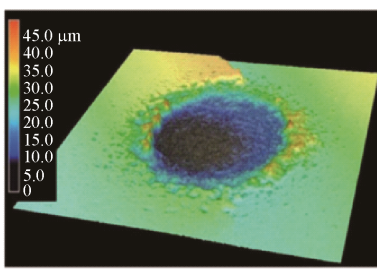

(a) 普通射流抛光

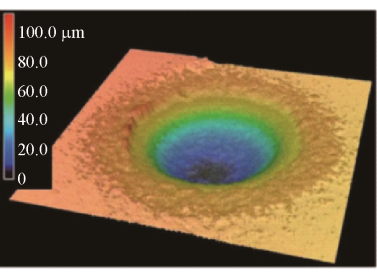

(b) 超声射流抛光
图 $12 \mathrm{SiC}$ 薄膜超声振动磨料射流加工

\section{5 超声振动柔性垫流体抛光}

柔性垫流体抛光是单晶硅基片、光学镜片等零 件表面抛光的主要方法。在此基础上，对抛光垫或 工件施加超声振动, 形成了超声振动柔性垫流体抛 光。在边界润滑摩擦状态下(图 13), 在多孔隙的抛 光垫与工件表面接触部位, 带动磨粒对工件表面进 行振动挤压、刻划与微切削去除; 在非接触部位, 超声振动驱动磨料流对工件表面产生冲击和空化去 除。两者共同作用, 完成抛光加工。

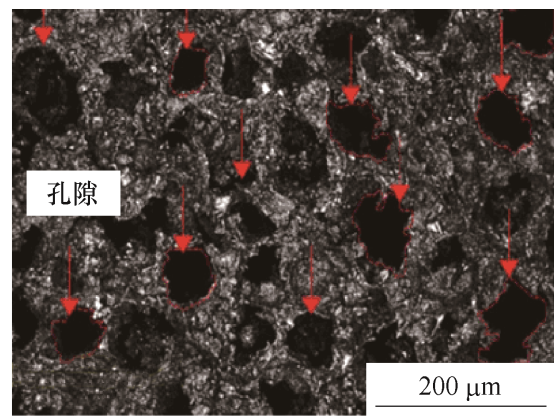

(a) 抛光垫显微结构

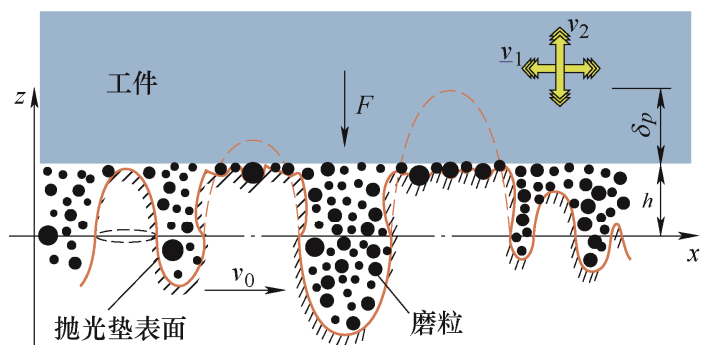

(b) 接触状态

图 13 抛光垫微观结构及其与工件表面的接触状态

SUZUKI 等 ${ }^{[52-53]}$ 研制了频率为 $25 \mathrm{kHz}$ 单向超声 振动辅助抛光系统(图 14a) 和频率为 $29 \mathrm{kHz}+22 \mathrm{kHz}$ 的两维超声振动辅助抛光系统(图 14b), 应用于 WC 
微型非球面模具的抛光。采用聚氨酯抛光工具, 纳 米级金刚石微粉抛光液, 在微载荷 $(2 \mathrm{mN})$ 下, 与五 轴数控机床相配合, 对非球面微镜片模具曲面进行 抛光。分别取得了粗粘度 $R y 7 \mathrm{~nm}$ 和 $R z 8 \mathrm{~nm}$ 的表面 质量。韩否等 ${ }^{[54]}$ 开展了单晶硅基片轴向超声振动抛 光试验, 应用碳化硅磨料和二氧化硅抛光液进行了 对比试验。结果表明, 采用超声振动柔性垫流体抛 光技术能够有效地提升材料去除率, 降低材料表面 粗䊁度, 改善材料加工表面形貌, 保证平面度。

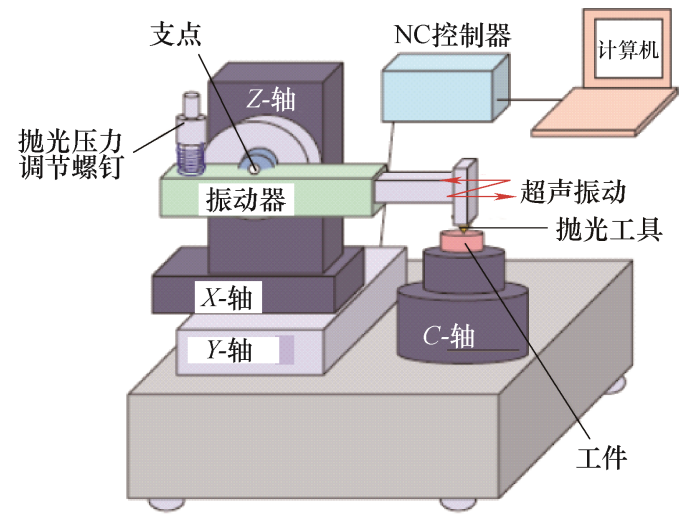

(a) 切向振动抛光

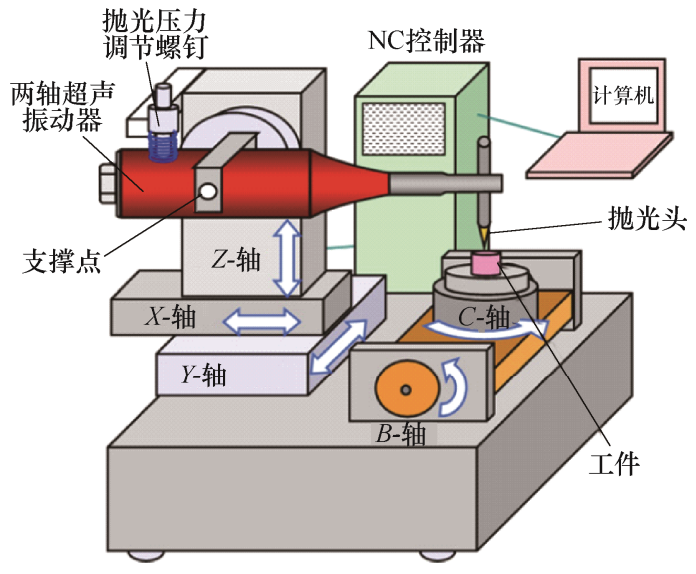

(b) 两维振动抛光

图 14 超声磨料流柔性抛光加工

$\mathrm{YU}$ 等 ${ }^{[55]}$ 开展了柔性抛光头超声振动复合抛光 光学玻璃透镜的研究(图 15), 采用超声雾化供液。 抛光光学玻璃镜片的材料去除率明显提高, 抛光 表面粗糙度可达 $R a 2 \mathrm{~nm}$; 材料去除率随着磨粒粒 径, 抛光头转速和超声波振幅的增加而显著增加, 可达普通抛光的两倍。赵清亮等 ${ }^{[6-57]}$ 开发了基于 工件超声振动的 $35 \mathrm{kHz} 、 25 \mathrm{kHz}$ 试验装置(图 16), 对 $\mathrm{SiC} 、 \mathrm{WC}$ 材料表面微圆弧槽阵列开展了超声振 动抛光的研究, 选用聚合物抛光轮和氧化铈磨料 抛光液对圆弧槽阵列进行抛光, 得到了理想的抛 光效果。有效地去除了硬脆材料微结构亚表面的 微裂纹, 微结构的表面粗鋉度小于 $R a 20 \mathrm{~nm}$, 锐 边尖角圆弧半径小于 $5 \mu \mathrm{m}$ 。材料去除率也明显高
于普通抛光, 加工效果随着频率和振幅的增高而更 加明显。

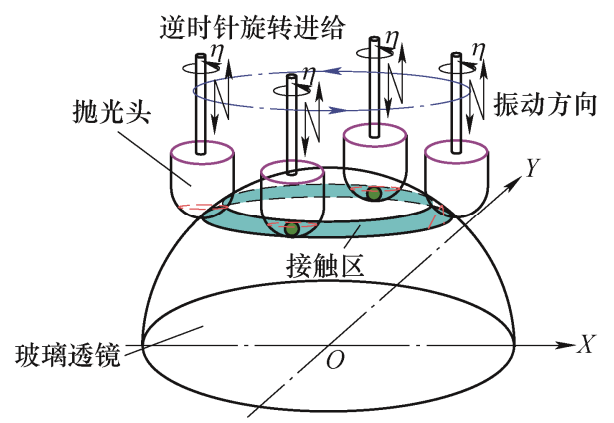

图 15 超声振动抛光透镜

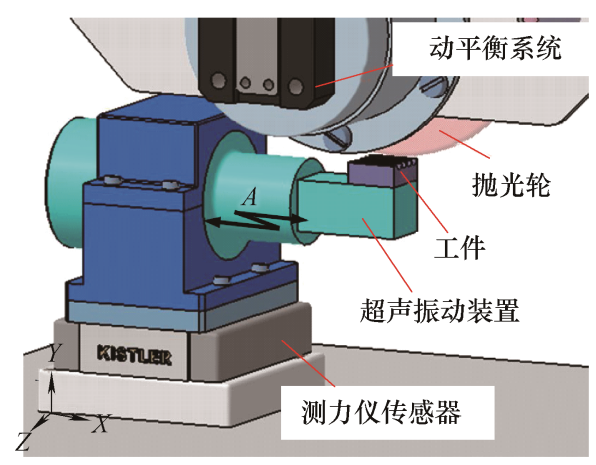

图 16 微结构超声振动抛光

\section{6 超声振动化学机械抛光}

在传统的化学机械抛光(Chemical mechanical polishing, CMP)方法基础上对工件或抛光垫施加超 声振动, 产生了超声振动 $\mathrm{CMP}$ 。

超声振动 $\mathrm{CMP}$ 是超声振动、流体动力和化学 反应复合作用的过程。施加超声振动不仅增强了磨 粒抛光的机械作用, 对于化学反应也具有明显的促 进作用。目前主要用在单晶硅、蓝宝石等基片的抛 光加工中。以单晶硅基片的高效抛光为目标, 把超 声振动与 CMP 相结合, 开展基础和应用研究是近 年来的研究热点之一。

$\mathrm{XU}$ 等 ${ }^{[59-60]}$ 应用聚氨酯多孔抛光垫, 分别采用 水平方向超声振动和圆盘弯曲超声振动两种方式对 蓝宝石工件开展了超声振动 CMP 试验。结果表明: 在同样条件下, 拋光表面质量和材料去除率都高于 传统 CMP。超声振动抛光表面粗粘度达到 $R a 0.083 \mathrm{~nm}$, 表面平整度达到 $0.12 \mu \mathrm{m}$, 材料去除率 达到 $22.8 \mathrm{mg} / \mathrm{h}$, 比普通的 CMP 提高 1 倍多。

何就等 ${ }^{[61-63]}$ 开展了单晶硅基片超声振动 $\mathrm{CMP}$ 系列研究, 先后研发了兆赫超声 $(1.7 \mathrm{MHz})$ 抛光装置 (图 17)和 $50 \mathrm{kHz}$ 圆盘端面椭圆振动抛光装置, 开展 了单晶硅片平面和边缘的抛光试验。试验表明：同 样条件下的表面粗粘度有明显降低, 兆赫超声抛光 后的硅片表面粗粘度均值可达到 $R a 0.031 \mu \mathrm{m}$ 。 


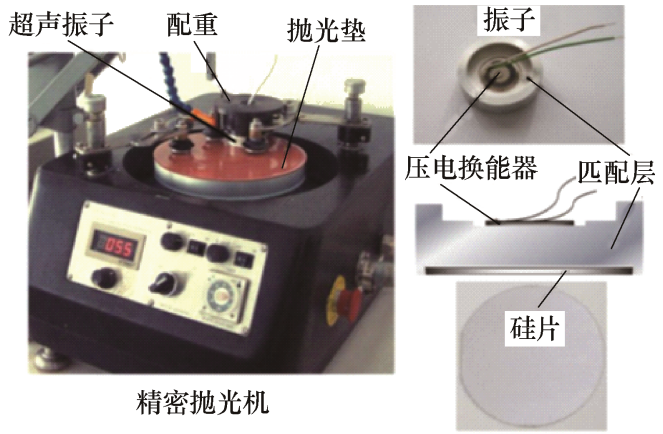

图 17 兆赫超声振动化学机械抛光

LIU 等 ${ }^{[64]}$ 研制了纵弯复合椭圆超声振动系统, 开展单晶硅椭圆超声振动 CMP 研究(图 18)。选用 $50 \mathrm{~nm}$ 的 $\mathrm{SiO}_{2}$ 磨料化学抛光液进行了传统 CMP、垂 直方向超声振动 CMP、水平方向超声振动 CMP、 椭圆超声振动 CMP 四种方法的对比试验, 结果表 明超声振动 CMP 的加工表面质量和材料去除率都 明显高于传统化学机械抛光方法。在采用椭圆振动 抛光时, 材料去除率比传统 CMP 高两倍, 抛光表 面粗糙度可达到 Ra10.61 nm(图 19)。

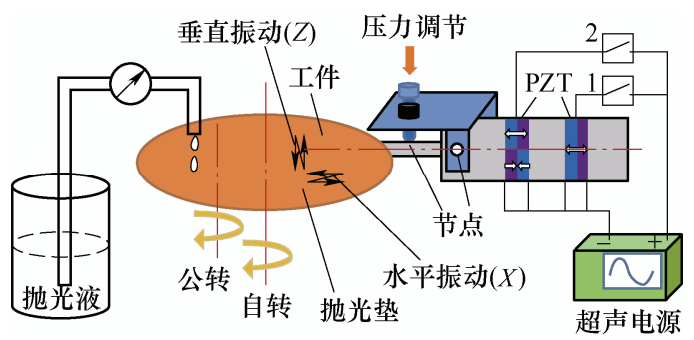

图 18 单晶硅椭圆超声振动化学机械抛光

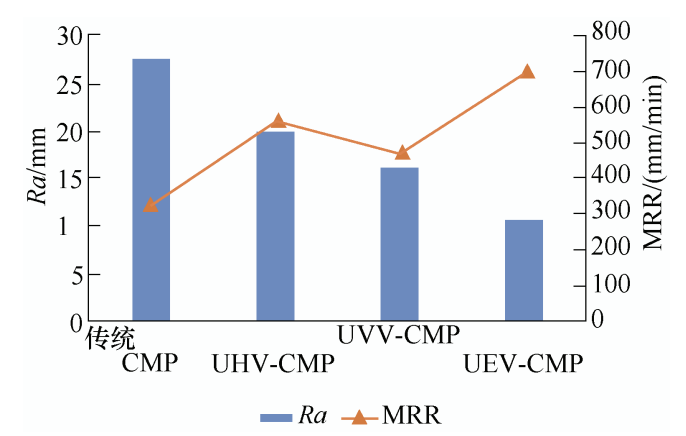

图 19 不同方式超声振动 CMP 效果对比

TSAI 等 ${ }^{[58]}$ 开展了铜基片的超声振动 CMP 与普 通 CMP 的比较试验(图 20)。试验表明, 超声振动增 强了铜片表面所受的机械及化学作用, 材料去除率 比普通 CMP 提高 $50 \%$ \% $0 \%$, 抛光扭矩减小了 $60 \%$ 。表面粗粘度达到 $R a 1.448 \mathrm{~nm}$ 。

翟文杰等 ${ }^{[66-67]}$ 开展了 $\mathrm{SiC}$ 陶瓷超声振动电化学 机械抛光研究(图 21)。结果表明, 超声振动有助于 提高电化学机械抛光过程的抛光质量和材料去除 率。使用聚氨酯抛光盘时, $\mathrm{SiC}$ 试件材料去除率可 达到 $3.57 \mu \mathrm{m} / \mathrm{h}$, 表面粗糙度 $R a 34.8 \mathrm{~nm}$; 相对于无
超声振动时，试件材料去除率提高了 $88.9 \%$ 。

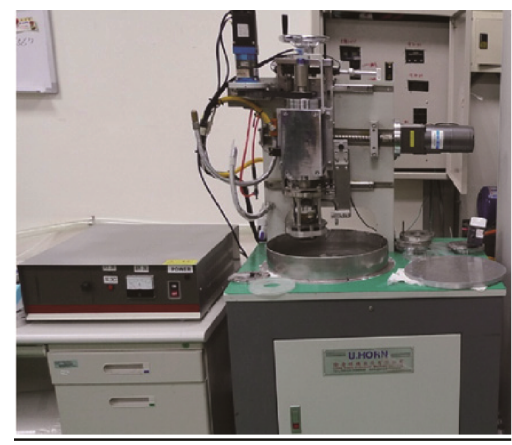

(a) 抛光设备

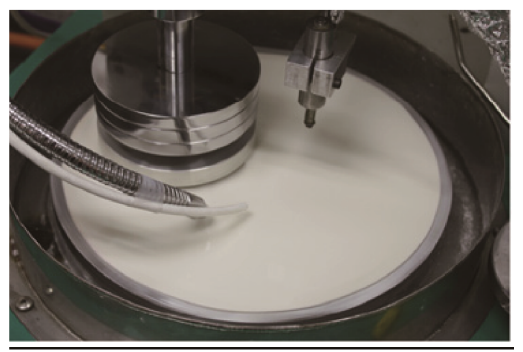

(b) 抛光加工

图 20 铜基片超声振动化学机械抛光

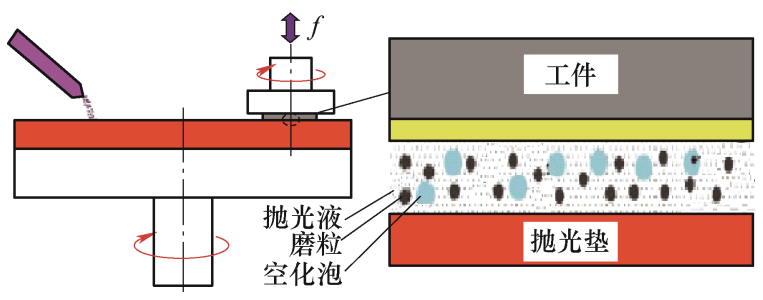

图 $21 \mathrm{SiC}$ 陶瓷超声振动电化学机械抛光

KOBAYASHI 等 ${ }^{[65]}$ 开发了用于硅片边缘抛光 的超声椭圆振动 CMP 系统(图 22)。抛光垫在硅片径 向平面内作椭圆超声振动。选用聚合物抛光垫材料, 水基 $\mathrm{SiO}_{2}$ 磨料抛光液, 进行了抛光试验。由于超声 椭圆振动的作用, 磨料对工件的冲击作用明显增强, 抛光效率明显提高; 椭圆振动使抛光垫与工件表面 之间能保持液体的流动, 实现了可靠的流体抛光作 用, 硅片边缘抛光表面粗粘度比普通 CMP 降低 $31 \% \sim 37 \%$, 抛光表面形貌也得到较大的改善。

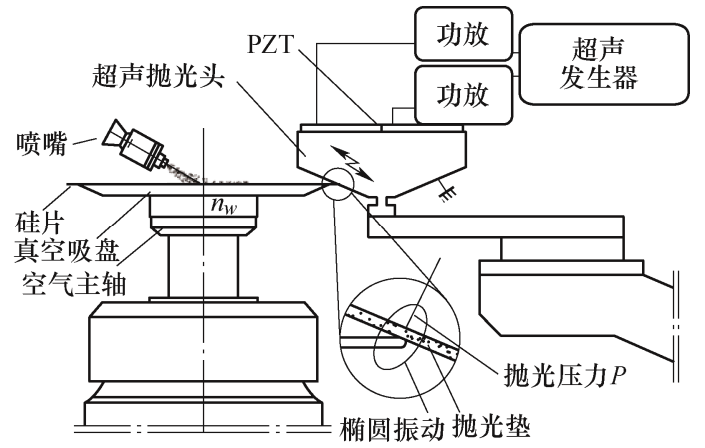

图 22 硅片边缘栯圆超声振动 CMP 


\section{UVAFP 材料去除机理研究}

\section{1 材料去除机理研究}

(1) 流体超声振动时的去除机理。

ICHIDA 等 ${ }^{[26]}$ 提出了磨料流超声振动时三种作 用模式的去除机理, 如图 23 所示。一是流体中空化 泡溃破射流在工件表面造成的去蚀。这种作用会在 工件表面留下的圆形去蚀坑; 二是由于空化泡溃破 使磨粒加速冲击, 在工件表面上碰撞或滑动而导致 的材料去蚀。这种作用会在工件表面上留下楔形的 凹坑或划痕。其数量要多于前一种; 三是由超声振 动驱动流体中磨粒对工件表面直接冲击或划擦, 实 现纳米级材料去除, 形成去蚀痕迹。在三种模式中, 第一种去除量最大, 第三种最小 (表 1)。要实现高光 滑的加工表面, 就要尽量避免超声空化造成的前两 种去除模式产生，设法增大第三种作用模式的效果。 但作者在研究中没有考虑工具与工件表面的距离、 超声振动频率以及工件材料不同对三种作用模式 的影响。

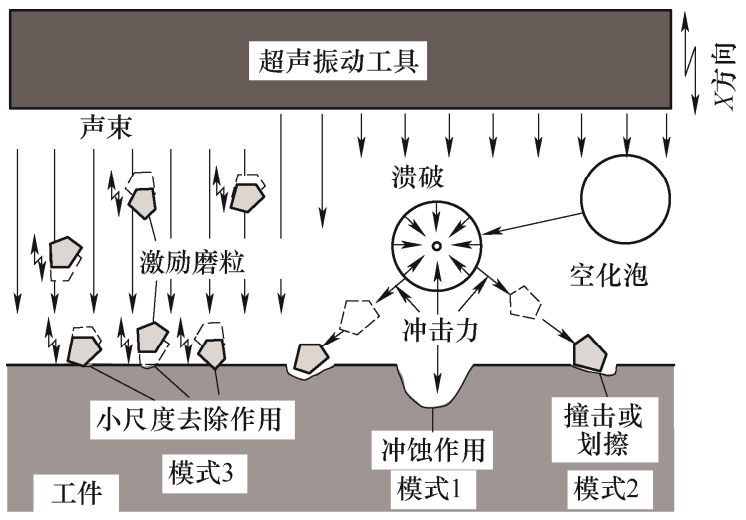

图 23 UVAFP 加工磨粒的作用模式

表 1 UVAFP 加工磨粒的作用模式特征

\begin{tabular}{cccc}
\hline \multirow{2}{*}{ 加工 } & \multicolumn{2}{c}{ 空化溃破冲击力 } & 超声能量 \\
\cline { 2 - 4 } & 冲蚀 & 磨粒碰撞或滑动 & $\begin{array}{c}\text { 磨粒激励小尺 } \\
\text { 度去除 }\end{array}$ \\
\hline 大小 & $\varphi 0.5 \sim 5 \mu \mathrm{m}$ & $0.3 \sim 1.5 \mu \mathrm{m}$ & $40 \sim 60 \mathrm{~nm}$ \\
深度 & $0.05 \sim 1.0 \mu \mathrm{m}$ & $0.01 \sim 0.3 \mu \mathrm{m}$ & $3 \sim 6 \mathrm{~nm}$ \\
形状 & 圆形侵蚀坑 & 楔形凹坑或划痕 & 纳米级痕迹 \\
\hline
\end{tabular}

TAN K 等 ${ }^{[68-69]}$ 指出, 在粗粘表面的低谷、缝隙 等部位易夹带气体, 形成产生空化核。在超声振动 作用下该空化核长大和溃破, 作者称之为异质空化。 在超声振动磨料流中, 空化泡溃破的微射流对被加 工表面的作用有两种形式(图 24): 一是微射流直接 作用于工件表面, 在工件表面的微凸峰处产生剪应
力, 去除工件表面的凸峰; 二是微射流压力通过 磨粒作用于工件表面, 产生法向去除和切向去除 作用, 去除表面的微观凸峰, 使表面微结构趋于 均匀光滑。

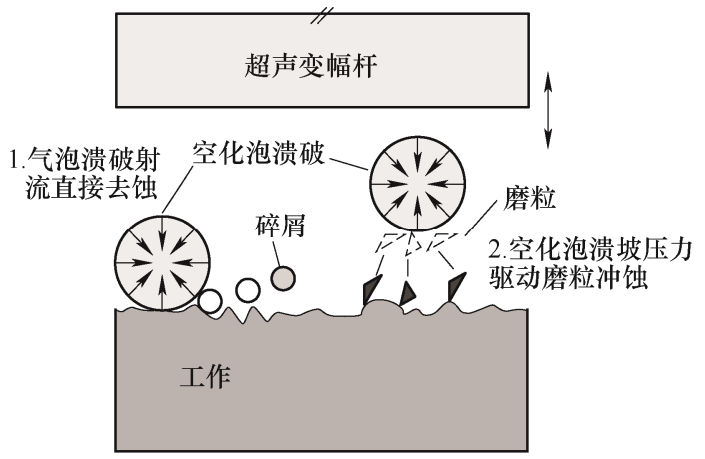

图 24 超声空化去除的两种作用机理

BEAUCAMP 等 ${ }^{[38-39]}$ 研究了聚焦超声磨料流抛 光时的去除机理。发现在聚焦超声磨料射流抛光时 空化作用和振动冲击作用产生的去除较小。而冲击 区域中磨料流体的横向速度周期性变化, 使工件表 面受到磨料流的剪切力作用是材料去除的主要 因素。ZAREPOUR 等 ${ }^{[70]}$ 研究了工件超声振动时单 颗磨粒冲击去除机理。工件作超声振动时, 磨粒对 工件表面的冲击速度是超声振动速度与空化泡溃破 的冲击波速度的叠加。应用 $50 \mathrm{kHz}$ 超声振动系统和 金刚石微粉抛光液对单晶硅 $(100)$ 晶面和熔融石英 表面进行单颗粒抛光试验发现(图 25): 脆性材料在 受到磨粒冲击时, 会首先在接触区域中发生塑性变 形，在颗粒-材料界面处的塑性变形产生大的拉应 力, 导致横向裂纹产生和扩展并最终导致材料去除。 控制磨粒的大小和超声振动的振幅可以控制从延性 去除到脆性去除的转变。在较小的磨料颗粒和较低 振幅时, 磨料冲击使工件表面产生塑性变形, 随着 磨料尺度和振幅增大, 变形转变为脆性破坏。当磨 粒平均尺寸为 $1 \mu \mathrm{m}$ 、振幅 $3 \mu \mathrm{m}$ 时产生纯延性去除 (图 25a); 当磨粒平均尺寸为 $3 \mu \mathrm{m}$ 、振幅 $4 \mu \mathrm{m}$ 时产 生纯脆性性去除(图 25b)。当采用 $3 \mu \mathrm{m}$ 磨粒和 $3 \mu \mathrm{m}$ 振幅时, 产生部分延性材料去除。基于这一结论, 可以通过控制工艺参数和加工条件来控制材料去除 的模式。

万宏强等 ${ }^{[35]}$ 研究了工具超声振动超声和工件 低频振动复合作用下抛光的材料去除机理。认为除 了具有 ICHIDA 所提出的三种作用机理外, 在工件 机械振动与工具超声振动共同作用下，磨粒还以一 定的速度对工件表面上进行刻划与刮擦，实现工件 表面材料的塑性去除。 


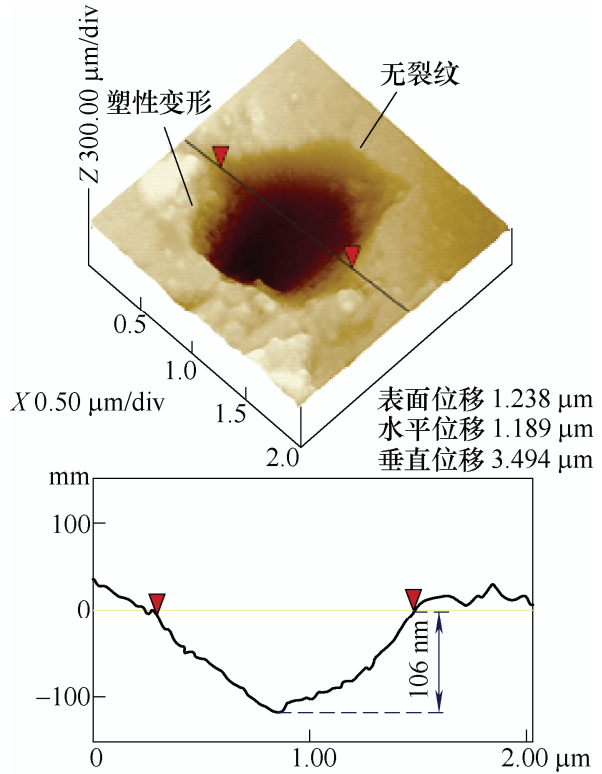

(a) 延性去除凹坑

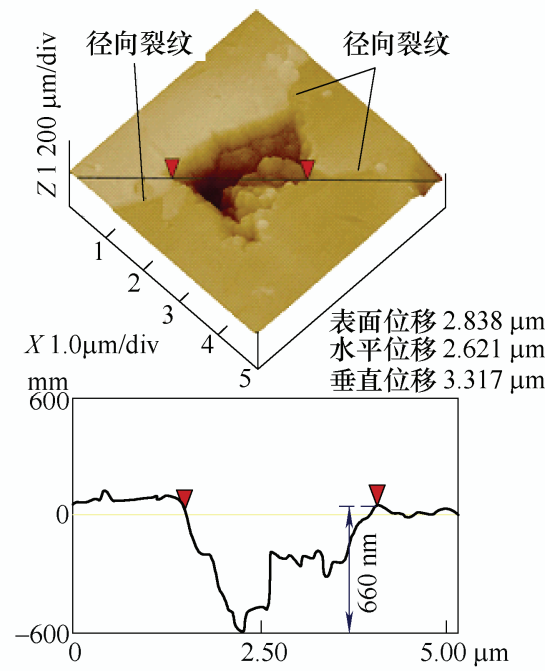

(b) 脆性去除凹坑

图 25 不同磨粒尺寸和振幅冲击凹坑的显微形态

(2) 超声振动射流抛光的去除机理。

如图 26 所示 ${ }^{[91,105]}$, 磨料射流抛光时, 射流垂 直入射时，工件表面射流中心点存在停滞区，射流 速度为零。在停滞去周围的环形区域内, 射流倾斜 冲击工件表面, 在垂直速度分量作用下, 磨料冲击 表面产生脆性或塑性压痕去除; 在切向分量作用下, 磨粒对工件表面耕犁或微切削去除。两种作用综合 形成截面呈 “W” 行的环形去蚀区。射流倾斜入射 时, 形成月牙形的去蚀区。

QI 等 ${ }^{[104-105]}$ 指出, 采用工件垂直(表面法线)方 向超声振动抛光, 可以减小 $\mathrm{W}$ 形横截面轮廓的深度 差。在一个振动周期内, 当工件正向移动接近喷嘴 时, 促进磨粒以较大的冲击角度冲击中心区域, 可 以增强磨粒对停滞区的动态冲击, 有助于去蚀区域 的均匀分布; 当工件反向移动远离喷嘴, 磨粒偏离
中心区域, 以较小的冲击角冲击工件表面, 产生高 效延性去除。获得相对平坦的冲蚀区表面(图 27)。

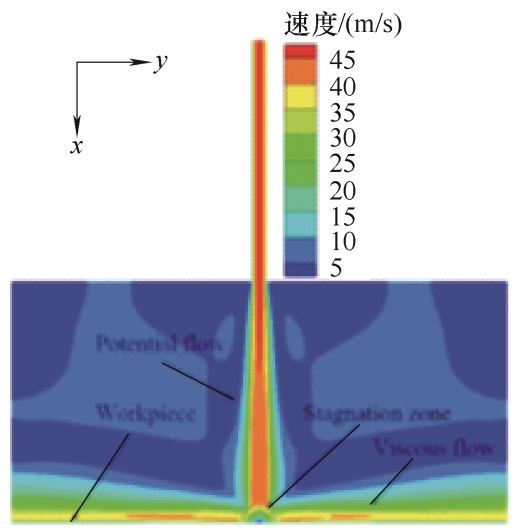

(a)

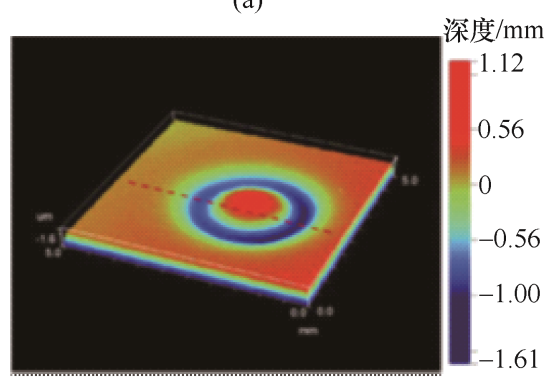

(b)

图 26 磨料射流垂直冲击去蚀特征

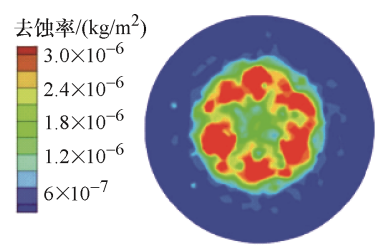

(a) 普通磨料射流

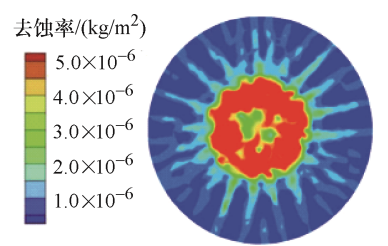

(b) 工件超声振动磨料射流
图 27 超声振动磨料射流去蚀率对比

LÜ 等 ${ }^{[49]}$ 研究了工件表面切向超声振动对停滞 区和侧流区冲击射流压力和速度的影响。结果表明: 随着振动振幅增加, 工件表面与液体边界层中的剪 切作用增强, 磨料流沿振动方向的侧流增强, 停滞 现象减弱。提高了磨粒的延性去除能力, 从而提高 抛光加工效率。

(3) 超声振动柔性垫抛光时的去除机理。

赵清亮 ${ }^{[57]}$ 、徐文虎等 ${ }^{[72]}$ 研究指出: 在超声振动 柔性垫抛光过程中, 抛光垫与工件表面的磨料流存 在两体磨粒磨损和三体磨粒磨损两种去除方式。在 超声振动作用下, 流体流动加快, 去除模式由两体 磨粒磨损向三体磨粒磨损转化。提高振动频率及振 动幅值有助于三体磨粒磨损比例的增加。同时, 超 声振动使抛光垫与工件表面的接触力增加, 嵌入磨 粒的运动轨迹增长并产生交错, 磨粒和工件表面之 间的化学反应增强; 孔隙中的磨料流在超声空化作 
用下对工件表面的冲击力增强, 促使工件表面摩擦 环境的变化, 表面反应边界膜的加速生成, 从而可 以获得更高的表面质量和去除率。

LIU 等 ${ }^{[64]}$ 、杨卫平等 ${ }^{[73]}$ 研究指出: 抛光工具做 超声椭圆振动时兼有超声纵向振动和超声切向振动 抛光的运动特性(图 28)。纵向超声振动带动磨料冲 击硅片表面氧化膜, 而切向超声振动将起到磨蚀、 撕裂硅片表面氧化膜的作用。两种振动的综合作用 加速磨粒硅片表面氧化膜的冲击、切削、腐蚀和破 坏, 有利于提高抛光效率。同时, 抛光工具周期性 超声椭圆振动导致抛光垫产生相应的变形, 使得抛 光垫与工件表面之间孔隙中的抛光液压力也发生变 化, 使工作区域中的抛光液始终处于流动状态 (图 29)。随着超声波振动幅度和频率的增加, 三体 磨粒磨损占比增大, 使磨料流均匀地冲击工件表面, 通过大量重叠的三体磨损作用去除工件表面的材 料。交变的冲击振动有利于抛光液磨粒细均匀分布, 并加速化学反应的速率, 提高抛光效率。研究结果 初步揭示了不同方式超声振动对材料去除机理的 影响。

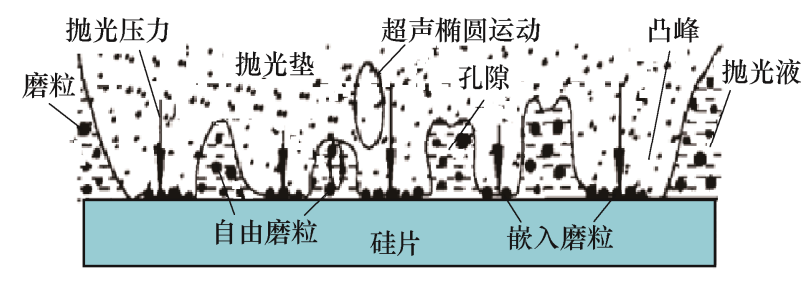

图 28 椭圆振动抛光模型

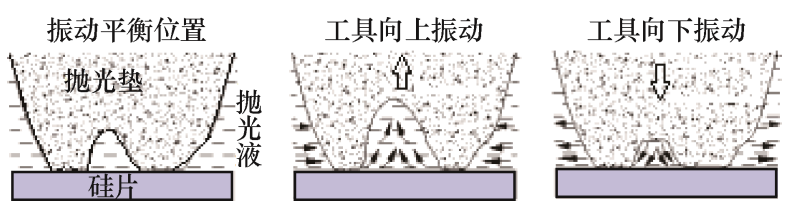

图 29 抛光液的横向流动

$\mathrm{YU}$ 等 ${ }^{[30]}$ 、XU 等 ${ }^{[60]}$ 研究了超声振动对自由磨 粒和嵌入磨粒作用, 在超声振动的作用下, 嵌入磨 粒的轨迹变为复杂的空间正弦曲线。切向振动改变 磨粒接触刻划运动轨迹, 增加超声振动频率和振幅 使磨粒的路径变长, 并增大磨粒的运动速度和加速 度, 提高抛光质量; 垂直方向振动使磨粒与工件表面 间歇接触, 可以降低平均微切削力和热量。在超声振 动和超声空化的作用下, 自由悬浮磨粒的瞬时速度和 加速度高频波动, 对工件表面产生冲击作用, 促进工 件表面微观粗粘凸峰的去除, 提高了抛光效率。

\section{2 流场特性研究}

(1) 超声振动时横向剪切流的研究。

$\mathrm{YU}$ 等 ${ }^{[29]}$ 、杨卫平等 ${ }^{[73]}$ 研究表明: 在超声振动
作用下, 工具与工件表面之间薄膜流场的压力和速 度周期性地变化, 与工件表面平行方向上的流场速 度大大增加, 而在在垂直与工件表面的方向上的流 场速度相对较低, 表明超声振动在流场中形成了强 烈的横向剪切流(图 30)。流体速度和压力随着超声 振幅的增加和流体膜厚度的减小而增加。

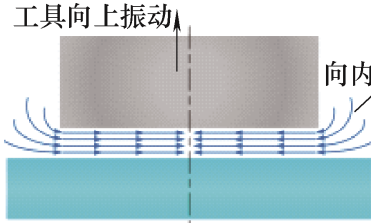

(a) 前半振动周期

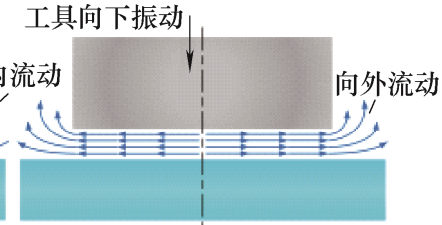

(b) 后半振动周期
图 30 流体层的横向剪切流

（2）流体膜厚度变化对流场的影响。

翟文杰等 ${ }^{[74-76]}$ 应用 FLUENT、COMSOL 等软件 仿真分析了工件超声振动时抛光垫与工件之间流体 膜厚度、抛光垫小孔参数等对流体薄膜压力、流体 速度等的影响。研究表明: 超声振动使磨料流的速 度、压力呈现正弦变化。当工件振动到波峰位置时, 流体膜最薄, 流场达到最大正压力, 入口速度为零。 在抛光垫与工件表面之间, 流场的压力、速度数值 由中线向两侧降低至零; 在特定的液膜厚度下超声 振动产生的纵波形成驻波, 可使试件表面处的机械 波振幅最大, 此时流场各特征参量取得最优值。抛 光垫上的孔隙使流场的速度、压力变化不连贯, 流 入孔隙的磨料液形成浴流, 使流体膜速度降低。

（3）超声空化对流场的影响。

吕婷等 ${ }^{[77]}$ 利用 FLUENT 模拟分析了工具超声 振动时工具端面与工件表面之间的流场内空化现象 对压力分布、流体速度等参数影响(图 31)。结果表 明: 在超声振动作用下, 流体中的空化周期性变化。 空化泡溃灭产生的冲击波提高了工件表面流体速度 和流场压力。在工件表面中心处的压力和速度最小, 中心周围压力和速度迅速增大, 然后沿径向缓慢减 小。压力的变化, 又促进了空化泡的产生和溃灭, 从而进一步影响流体的速度。

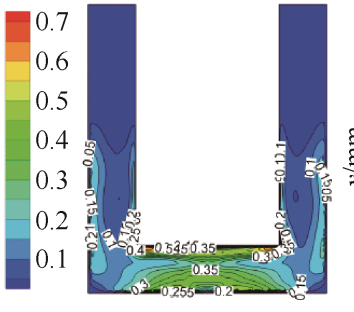

(a) 速度分布

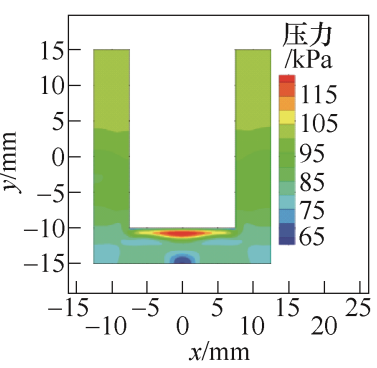

(b) 压力分布
图 31 流场内速度与压力分布 
超声频率在一定范围内增高时, 流场内部绝对 压力差值变大, 有利于空化产生, 从而增大工件表 面的流速。但是当频率超过一定值后, 由于超声振 动频率过高, 空化泡的形成时间缩短, 来不及发生 崩溃, 使得空化作用有所削弱, 会降低工件表面的 流体速度。超声振幅的增大, 会使流场绝对压力以 及压力差值增大, 易产生空化, 使得磨粒瞬时速度 增大。工具与工件表面之间的距离越近, 会使超声 振动产生的能量直接作用于工件表面的比例越大, 同时也使得空化泡产生和溃灭的周期缩短, 促使工 件表面的磨粒速度提升。

$L U ̈$ 等 $^{[49]}$ 基于完全空化模型, 利用 CFD 数值模 拟分析了工件切向超声振动射流抛光过程中的空化 现象。结果也表明, 在射流冲击过程中, 空化泡在 工件表面周期性地产生和圾塌, 空化气泡的演变在 很大程度上取决于压力和湍流的变化。增加超声振 幅时, 空化作用增强; 超声频率升高时, 空化作用 减小。工件切向超声振动可以使沿振动方向的横向 流动增强, 速度增高。

(4) 超声振动对湍流的影响研究。

计时鸣等 ${ }^{[33-34,78]}$ 分析了加载超声波激振的磨料 流在约束流道内的速度和湍动能。研究表明: 超声 振动强化了磨料流的湍流状态, 使流体湍动能的分 布更均匀, 速度和压力变化更加剧烈。由于湍流流 体形态无序化, 磨料颗粒作无序化运动, 使工件表 面纹理无序化。空化气泡溃破更增加了湍流, 使工 件的复杂形腔表面得到均匀的抛光。

\section{UVAFP 材料去除模型研究}

在磨料流抛光过程中, 根据工件材料特性和冲 击强度的不同, 工件表面存在冲击塑性变形、微切 削、脆性破碎几种形式。这几种形式都会造成工件 表面的微观形貌变化, 因而都属于抛光时的材料去 除, 分别可称为塑性变形去除、微切削去除和脆性 破碎去除。

在传统抛光的理论研究中, 主要是以 Herzt 接 触理论为基础, 应用压痕力学分析单颗磨粒冲击工 件表面产生的变形去除。国内外学者对此有大量的 研究 ${ }^{[79-85]}$ 。对于 UVAFP 过程的理论研究, 也是在 接触理论基础上, 应用分子动力学模拟、SPH 方法、 有限元分析和试验等方法, 开展超声振动作用下单 颗磨粒冲击工件表面的材料去除理论研究, 建立理 论模型, 揭示超声振动对脆性和延性去除模式转变 的影响。
在流体动力和超声振动的作用下, 磨粒以一 定的角度对工件表面冲击(图 32)。设磨料流的流 速为 $v_{L}$, 质量为 $m_{p}$ 的磨粒沿与工件表面的夹角 为 $\theta$ 的方向冲击工件表面, 振动方向与冲击方向 相同。工具端面上的振幅为 $A$, 振动频率为 $f$, 磨 料流的密度为 $\rho_{0}$, 工具振动端面处的液体声压为 $p_{0}$, 衰减系数为 $\alpha$, 声速为 $c$, 工具振动端面到 工件表面的距离为 $h$, 则磨粒冲击工件表面时的 速度幅值 ${ }^{[86]}$ 为

$$
v_{r}=v_{L}+\frac{p_{0}}{\rho_{0} c} e^{-\alpha h}
$$

式中, $v_{r}$ 的垂直分量和水平分量分别时 $v_{m n}$ 和 $v_{m t}$ 。

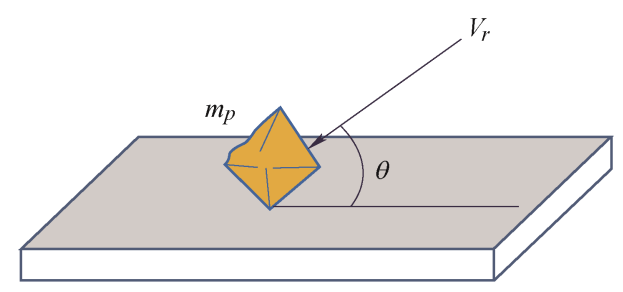

图 32 磨粒对表面的冲击

\section{1 单颗磨粒冲击塑性材料的去除模型}

(1) 垂直冲击。

当 $\theta=90^{\circ}$ 时, $v_{r}=v_{m n}$ 。根据 Herzt 接触理论, 磨粒垂直冲击塑性材料是一个由弹性变形到塑性变 形的过程, 冲击开始时首先产生弹性变形, 当应力 达到工件材料的屈服极限时，产生屈服变形。磨粒 速度为 $v_{m n}$ 时的冲击力 $P$ 为 ${ }^{[87]}$

$$
P=\frac{4}{3}\left(\frac{5 \pi}{4} \rho\right)^{\frac{3}{5}}\left(\frac{1-v_{1}^{2}}{E_{1}}+\frac{1-v_{2}^{2}}{E_{2}}\right)^{-\frac{2}{5}} R^{2} v_{m n}^{\frac{6}{5}}
$$

式中, $R$ 为磨粒半径; $v_{1}$ 和 $v_{2}$ 分别为磨粒和工件材 料的泊松比; $E_{1}$ 和 $E_{2}$ 分别为磨粒和工件材料的弹性 模量； $\rho$ 为磨粒的材料密度。

当磨粒冲击光滑工件表面时, 冲击压入深度 $\lambda$ 为

$$
\lambda=\left[\frac{9}{16} \frac{P^{2}}{R}\left(\frac{1-v_{1}^{2}}{E_{1}}+\frac{1-v_{2}^{2}}{E_{2}}\right)^{2}\right]^{\frac{1}{3}}
$$

冲击产生的塑性变形去除体积 $V_{S}$ 为 ${ }^{[88]}$

$$
V_{s}=\frac{5 \pi}{4} \varsigma_{c} \rho\left(\frac{1-v_{1}^{2}}{E_{1}}+\frac{1-v_{2}^{2}}{E_{2}}\right) R^{2} v_{m n}^{2}
$$

式中, $\zeta_{c}$ 为考虑弹性变形恢复等因素的冲击变形 系数。

当工件表面存在微观凸峰时, 磨粒首先冲击这 
些凸峰。当冲击应力达到工件材料的屈服极限时, 凸峰产生塑性变形。由动力学方程可以求得塑性变 形深度 $\lambda_{s}$ 为

$$
\lambda_{s}=\frac{\frac{4 \pi \rho R^{3} v_{m n}^{2}}{3 S_{s} \sigma_{s}}-4 \sqrt{2 R} \lambda_{1 \max }{ }^{\frac{3}{2}}}{\left[15 S_{s} \sigma_{s}\left(\frac{1-v_{1}^{2}}{E_{1}}+\frac{1-v_{2}^{2}}{E_{2}}\right)\right]}
$$

式中, $\lambda_{1 \max }$ 为最大弹性变形量; $S_{1}$ 为产生塑性变形 时磨粒与凸峰的接触面积; $\sigma_{s}$ 是工件材料的屈服极 限。显然, 凸峰的塑性变形去除量与 $\lambda_{s}$ 成正比, 也 即与磨粒的大小、密度和冲击速度成正比。

\section{(2) 倾斜冲击。}

磨粒倾斜冲击塑性材料时, 在水平方向对工件 表面进行微切削、刻划作用(图 33), 使工件表面产 生塑性变形去除和微切削去除。当工件表面为光滑 表面时, 张成光等 ${ }^{[88]}$ 在 Finnie 理论模型基础上, 经 过试验修正, 得到去除量

$$
V_{Q}=\left\{\begin{array}{l}
\frac{m_{p} v_{r}^{2.4}}{8 p}\left(\sin 2 \theta-3 \sin ^{2} \theta\right) \quad \theta \leqslant 18.5^{\circ} \\
\frac{m_{p} v_{r}^{2.4}}{8 p} \cos ^{2} \theta \quad \theta>18.5^{\circ}
\end{array}\right.
$$

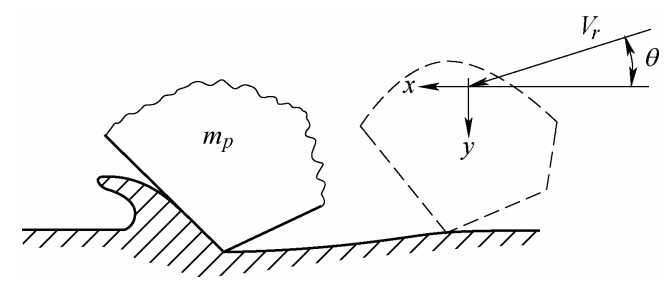

图 33 倾斜冲击塑性材料的切削与耕犁

ANTHONY 等 ${ }^{[38]}$ 在此基础上, 考虑超声振动的 作用, 推导出单颗磨粒的去除体积

$$
\begin{gathered}
V_{q}=k_{q}\left[\frac{1}{2} m_{p}\left(v_{m t}{ }^{2}+\frac{1}{2} v_{m t z}{ }^{2}\right)\right] \times \\
{\left[\frac{1}{2} m_{p}\left(v_{m n}{ }^{2}+\frac{1}{2} v_{m n z}{ }^{2}\right)\right]^{\frac{2(1-b)}{3}}}
\end{gathered}
$$

式中, $k_{q}$ 为与工件材料塑性流动和弹性恢复有关的 常系数, $b$ 为截面指数。 $v_{m t z} 、 v_{m n z}$ 分别是磨粒超声 振动的切向和法向速度分量。

\section{2 单颗磨粒冲击硬脆性材料的去除模型}

(1) 垂直冲击。

如图 34 所示, 当磨粒垂直冲击硬脆性工件表面 时, 经历了弹性变形-塑性变形-脆性破坏的过程。当 冲击应力达到屈服极限时, 压痕产生塑性变形 ${ }^{[89]}$,

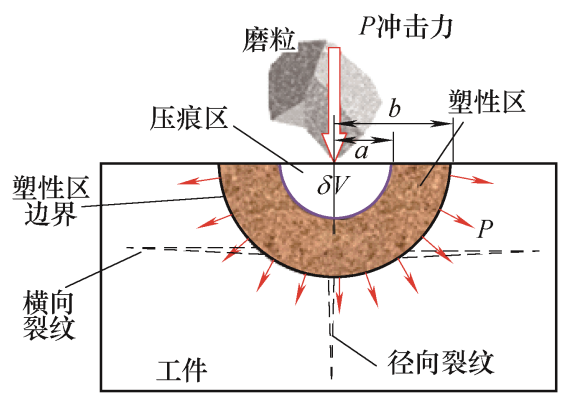

图 34 脆性材料冲击压痕和变形区

其压痕深度为

$$
\begin{aligned}
& \lambda_{s}=\frac{1}{\sigma_{s} S_{s}}\left[\frac{2}{3} \pi \rho R^{3} v_{m n}{ }^{2}-\right. \\
& \left.\frac{8}{15}\left(\frac{1-v_{1}^{2}}{E_{1}}+\frac{1-v_{2}^{2}}{E_{2}}\right)^{-1} R^{\frac{1}{2}} \lambda_{Y}{ }^{\frac{5}{2}}\right]
\end{aligned}
$$

式中, $\lambda_{y}=\frac{\sigma_{s} d}{E_{2}}, d$ 为工件的厚度。压痕体积 $\delta V$ 与 冲击力 $P$ 的关系为 ${ }^{[80]}$

$$
\delta V=\frac{1}{3} \cot \psi\left[\frac{P}{H \alpha^{\frac{1}{3}}}\right]^{\frac{3}{2}}
$$

式中, $H$ 是工件材料的硬度， $\psi$ 是磨粒底边的夹角。 $a$ 是磨粒形状因子。

由 ZAREPOUR 等 ${ }^{[89]}$ 研究可知, 当冲击应力大 于脆性破坏临界值时, 压痕周围产生裂纹。产生横 向裂纹时压痕的临界体积 $\delta V_{L}{ }^{*}$ 和产生径向裂纹时 压痕的临界体积 $\delta V_{R} *$ 分别为

$$
\left\{\begin{array}{l}
\delta V_{L}^{*}=\frac{1}{3} \frac{\varsigma_{0}^{\frac{3}{2}}}{\alpha^{\frac{1}{2}} A^{3}} \frac{E_{2}^{\frac{3}{2}} K_{c}^{6}}{H^{\frac{15}{2}}} \\
\delta V_{R}^{*}=\left(\frac{3}{2 \pi}\right)^{3} \frac{\mu^{6}}{\beta^{6}} \frac{E_{2}^{\frac{3}{2}} K_{c}^{6}}{H^{\frac{15}{2}}}
\end{array}\right.
$$

式中, 常数 $\zeta_{0}=1.2 \times 10^{3}, A=0.75$ 是压痕形状系数。 $K_{c}$ 是工件材料的断裂强度极限。 $\mu=0.63$ 是反映压 痕与塑性区大小关系的常数, $\beta=0.096$ 是与工件材 料和压头形状无关的常数 ${ }^{[89]}$ 。

(2) 倾斜冲击。

对于粗糙表面, 在抛光的初期, 磨粒倾斜冲击 表面上的凸峰，使凸峰产生剪切破坏，从而降低高 度，当表面高度趋于一致后，磨粒对表面的作用逐 步转变为对光滑表面冲击去除方式。

吕哲等 ${ }^{[99]}$ 提出，磨粒倾斜冲击脆性材料时，对 于光滑表面, 冲击动能的法向分量 $U_{m n}$ 与工件材料 产生脆性破坏的临界动能的关系决定了表面材料去 除的方式。材料产生脆性破坏的临界动能为 ${ }^{[89]}$ 


$$
U_{C}=C \frac{E^{\frac{3}{2}} K_{C}^{6}}{H^{\frac{13}{2}}}
$$

当 $U_{m n} \leqslant U_{C}$ 时, 工件表面以塑性变形方式去除。 当 $U_{m n}>U_{C}$ 时,材料产生脆性破坏,表面材料以脆性方 式去除，单个磨粒去除体积为

$$
V_{C}=f_{1} \frac{E_{2}^{\frac{5}{4}} U_{m n}^{\frac{7}{6}}}{H^{\frac{17}{12}} K_{C}}\left(1-f_{2} \frac{E_{2}^{\frac{1}{4}} K_{C}}{H^{\frac{13}{12}} U_{m n}^{\frac{1}{6}}}\right)
$$

通过对 SPH/FEM 组合建立的工件超声振动时 磨料射流抛光冲蚀去除模型研究发现, 在冲击角度 相同时, 超声振动条件下的磨料粒子冲蚀划痕深度 较大, 磨料颗粒与工件表面的接触力更大, 接触的 持续时间增加, 冲蚀速率高于普通磨料射流; 冲蚀 速率随着冲击角的增加而增加。在 $90^{\circ}$ 的冲击角度 下, 冲击力最大, 主要是脆性去除, 有最大冲蚀速 率; 在 $30^{\circ}$ 的冲击角度下, 尖锐多面体颗粒侵蚀速 率最大。主要为延性去除。

\section{3 磨料流束冲击去除模型}

在实际抛光过程中, 抛光表面是磨料流中大量 磨粒随机重叠冲击作用的结果。由于磨料流中磨粒 随机分布, 流体的流速和超声振动分布不均匀, 工 件表面同一位置受到多磨粒重叠或部分重叠冲击的 状态也不相同。这种状态影响加工表面的材料去除 率和表面粗粘度。在已有的文献中, 对磨料流束的 去除模型研究较少。

鲍慧雪等 ${ }^{[101]}$ 研究了工件表面上同一点被多个 磨粒重复冲击脆性材料的去除量。同一位置经多磨 粒重 $\mathrm{k}$ 次复冲击后, 累计去除材料量

$$
\begin{gathered}
M R=\sum_{i=1}^{k} M_{i} V_{i} \\
V_{i}=\pi\left(\sum_{m=0}^{i} \lambda_{m}\right)^{2}\left(R-\frac{1}{3} \sum_{m=0}^{i+1} \lambda_{m}\right)-\pi\left(\sum_{m=0}^{i-1} \lambda_{m}\right)^{2} \times \\
\left(R-\frac{1}{3} \sum_{m=0}^{i-1} \lambda_{m}\right)
\end{gathered}
$$

式中, $M_{i} 、 \lambda_{i}$ 分别为第 $i$ 次冲击的质点数、压痕深 度。 $V_{i}$ 为第 $i$ 次冲击后的去除量。

马立等 ${ }^{[102-103]}$ 应用接触理论推导了超声振动抛 光塑性材料时多颗磨粒重复作用时的塑性变形深度 $\lambda_{Y N}$ 和接触面积 $S_{N}$ 。在磨粒的重复作用下, 每次冲 击产生的塑性形变量逐渐减小。随着表面粗粘度降 低, 抛光速度逐渐降低。磨料流中多磨粒同时作用 时, 单位时间内去除体积为

$$
V_{A}=\frac{\rho f A R v_{m}^{2}}{10 \sigma_{S}} \chi^{\frac{2}{3}}
$$

式中, $A$ 为超声振动工具端面面积; $\chi$ 为流体中磨 粒的体积密度; $v_{m}$ 为冲击工件表面磨粒束的统计平 均速度。

上述研究是以单颗磨粒的去除模型为基础, 以 同一位置重复冲击为背景, 对单颗磨粒的冲击去除 简单叠加形成的去除模型。而实际的磨料流束冲击 过程要复杂得多, 对去除模型的研究尚需不断深入。

\section{5 结论}

(1) 各种 UVAFP 方法的比较。

根据超声振动在其中的作用不同, UVAFP 技术 些技术分为两大类, 即以超声振动能量为主的 UVAFP 方法和以超声振动与传统抛光方法结合的 复合 UVAFP 方法。

1) 超声振动磨料流冲击抛光、聚焦超声振动磨 料流抛光和超声振动磨料流场抛光三种方法属于第 一类方法。其中前者是利用工件与工具表面之间的 近距离法向振动, 使磨粒冲击工件表面, 实现材料 去除。这种方法主要适用于硬脆性材料的抛光, 相 对于传统的方法, 可以显著提高抛光效率。但表面 质量的提高能力不明显。为了高效地利用超声振动 的能量, 必须缩小工具与工件表面的距离, 也限制 了这一技术在曲面表面上的应用; 聚焦超声磨料流 抛光属于点抛光方法, 超声振动能量聚焦后, 可以 实现高效的抛光, 适合于曲面的轨迹法抛光。但是 聚焦超声产生的热效应会影响抛光后的表面组织; 最后一种方法是通过超声产生的紊流流场动能带动 磨粒对工件表面的去除，这种抛光方法兼具了磨粒 对工件表面的冲击和剪切作用, 可以用于硬脆材料 和金属材料超光滑表面的抛光，适应各种形状表面 的抛光, 但是受流场动能的限制, 其抛光效率不高。

2) 在复合 UVAFP 方法中, 超声振动磨料射流 抛光是点抛光方法, 在射流动能的基础上叠加超声 振动的动能使这种方法具有较高的抛光效率, 可以 适应硬脆性材料和金属材料抛光要求。其研究的热 点在如何通过工艺参数的优化, 实现高表面质量的 抛光。

超声振动 CMP 在硅片抛光中的应用是这一方法 的研究热点。硅片工件具有厚度小, 脆性大的特性, 抛光时要同时保证表面粗粘度和表面的平坦度要求, 在减薄加工时要求高效率。已有的研究采用超声振动 CMP 方法, 进行平面和边缘的抛光, 都取得了很好 的效果。同时, 这一方法也是光学镜片等内外曲面抛 光的有效方法。当前的研究主要集中在不同振动方式 
对抛光效果的影响以及工艺参数的优化研究。

各种 UVAMP 方法在不同抛光对象中的应用研 究情况见表 2。

(2) 材料去除机理与模型。

在超声振动激励下, 磨料流对工件表面的抛光 作用存在振动冲击、空化射流冲击、空化激励磨粒 冲击三种方式。其中后两种作用方式起主要去除作 用。抛光脆性材料时, 随着磨粒粒度和超声振幅的 增大，从延性去除向脆性去除的转变。

在超声振动和超声空化的作用下, 工件与振动 工具之间的流体中产生沿工件表面的强烈侧向流 动。侧向流动加速了磨粒的侧向速度, 磨粒对工件 表面的刻划和切削作用增强。磨料流中的磨粒以法 向和切向两种方式冲击工件表面。实现对工件表面
微观粗糙凸峰的去除。

以接触力学理论为基础, 已经形成了单颗磨粒 冲击时的去除模型。从实际加工过程看, 抛光过程 中磨粒对工件表面的冲击都可以分解为垂直和水平 两个方向, 垂直方向的冲击以产生塑性和脆性破坏 去除为主; 水平方向的冲击主要产生刻划、剪切去 除和微切削去除。对超声振动磨料流束的去除机理 研究还处在初始阶段, 已有的模型尚不能很好地表 达实际磨料流束的去除特征。

(3) 工艺系统研究。

超声振动辅助磨料流抛光技术必须要有相应的 振动抛光工艺系统才能实现。现有的研究大多是建 立在试验研究系统上, 这些试验系统与生产实际的 应用要求还有一定差距。

表 2 UVAMP 应用研究情况

\begin{tabular}{|c|c|c|c|c|}
\hline 工件 & 抛光方法 & 振动方式 & 振动参数 & 工艺效果 \\
\hline 硅片 & 超声振动 CMP & 工具或工件单向/椭圆振动 & $\begin{array}{l}20 \sim 40 \mathrm{kHz}, 30 \mathrm{~min} \\
1.7 \mathrm{MHz}\end{array}$ & $\begin{array}{l}R a \text { 从数百纳米降低到 } 20 \sim 30 \mathrm{~nm} \text {, } \\
\text { 抛光效率提高 } 30 \%\end{array}$ \\
\hline 硬脆材料 & $\begin{array}{l}\text { 超声振动磨料流 } \\
\text { 冲击抛光 }\end{array}$ & 工具纵向振动 & $20 \mathrm{kHz}$ & 效率和表面质量提高 \\
\hline 光滑表面 & $\begin{array}{l}\text { 超声振动磨料流 } \\
\text { 场抛光 }\end{array}$ & $\begin{array}{l}\text { 一维或两维阵列振动、驻波 } \\
\text { 声场 }\end{array}$ & $20 \sim 40 \mathrm{kHz}, 1 \sim 2 \mathrm{~h}$ 抛光 & 实现纳米级表面粗踈度 \\
\hline 金属内表面 & $\begin{array}{l}\text { 超声振动磨料流 } \\
\text { 挤压抛光 }\end{array}$ & 工件横向 /径向振动 & $\begin{array}{l}20 \mathrm{kHz} \text {, 振幅 } 10 \mu \mathrm{m} \\
6 \sim 15 \mathrm{~min} \text { 抛光 }\end{array}$ & 表面粗粘度降低 70\% \\
\hline 曲面 & $\begin{array}{l}\text { 超声振动柔性垫 } \\
\text { 流体抛光 }\end{array}$ & 工具单向/椭圆振动 & $20 \sim 30 \mathrm{kHz}$, 振幅 $19 \sim 20 \mu \mathrm{m}$ & $\begin{array}{l}\text { 表面粗糙度 } R a 1.2 \mathrm{~nm} \sim 4 \mathrm{~nm} \\
R y 7 \mathrm{~nm}\end{array}$ \\
\hline
\end{tabular}

\section{6 展望}

(1) 应深化超声振动对抛光过程的作用机理研 究。在现有的研究中, 超声振动对抛光过程的影响 只是简单地通过磨粒速度的增加来体现, 把超声振 动的振动速度幅值作为磨料流冲击速度的增量, 使 用传统的冲击机理来分析, 没有考虑交变应力作用 的影响。而实际上施加超声振动后磨粒对工件表面 的作用是流体动能与超声频脉动冲击的复合作用过 程, 冲击力是周期性脉动变化的。工件表面的同一 位置会受到不同磨粒的交变载荷冲击, 对于脆性材 料, 这种交变载荷冲击作用下的去除机理与传统的 动载荷冲击去除机理显然有所不同, 对工件表面的 作用和材料去除特征也不相同。因此, 研究有超声 振动时磨粒周期脉动冲击作用下的工件表面材料去 除机理是理解超声振动磨料流抛光机理本质的重要 基础, 应是今后这一技术基础研究的主要问题。

(2) 开展磨料流束去除模型和表面形成机理的 研究。在单颗磨粒去除模型研究的基础上, 结合不 同的抛光方法, 研究磨粒流束的去除机理和模型,
建立磨粒流参数、工艺参数、超声振动参数对材料 去除和表面成形影响的模型, 是基础研究问题之一。

(3) 研究聚焦超声振动磨料流抛光时的作用机 理, 揭示不同超声参数对材料去除影响的机理, 分 析影响抛光效果的主要因素。此外还应聚焦超声振 动抛光时的热效应开展研究, 热效应的存在会对工 件表面产生重要的影响。分析热作用的规律及其对 不同材料工件表面的影响是这一方法研究应关注的 问题。

(4) 开展工艺应用的基础研究。研究抛光工艺 参数、超声振动参数、磨料流特性对不同材料抛光 表面质量和抛光效率的影响。建立各种代表性的工 艺方法所适用的工艺范围、加工对象的规范; 建立 加工工艺参数、超声振动参数、磨料流参数等工艺 数据库。

(5) 研究超声振动方式对不同材料抛光的材料 去除和表面形成的影响。针对不同的振动方式(一维/ 两维)、不同的振动方向、不同的振动对象(工具/工 件)和不同的工件材料开展相应的机理研究, 从而建 立系统的理论。为技术的应用提供指导。

(6) 研究满足工程实际应用要求的工艺装备。 
目前的研究采用的装备大都是基于试验需要构建 的, 与工程应用有较大的差距。今后应加强各种超 声振动抛光工艺方法所需工艺系统的研究。开发出 符合生产实际需要的专用加工装置和系统, 推动精 密高效抛光技术的进步。

\section{参 考 文 献}

[1] 袁巨龙, 张飞虎, 戴一帆, 等. 超精密加工领域科学技 术发展研究[J]. 机械工程学报, 2010, 46(15): 161-177. YUAN Julong, ZHANG Feihu, DAI Yifan, et al. Development research of science and technologies in ultra-precision machining field[J]. Journal of Mechanical Engineering, 2010, 46(15): 161-177.

[2] 李敏, 袁巨龙, 吴喆, 等. 复杂曲面零件超精密加工方 法的研究进展[J]. 机械工程学报, 2015, 51(5): 178-191. LI Min, YUAN Julong, WU Zhe, et al. Progress in ultra-precision machining methods of complex curved parts[J]. Journal of Mechanical Engineering, 2015, 51(5): 178-191.

[3] 胡扬轩, 邓朝晖, 万林林, 等. 用于蓝宝石材料加工的 新型超精密抛光技术及复合抛光技术研究进展 $[\mathrm{J}]$. 材 料导报, 2018，32(9): 1452-1458.

HU Yangxuan, DENG Zhaohui, WAN Linlin, et al. Research progress of new ultra-precision polishing technology and composite polishing technology for sapphire material processing [J]. Materials Guide, 2008, 32(9): 1452-1458.

[4] 张辰阳, 王玉芬, 向在奎, 等. 石英玻璃表面精密加工 研究进展[J]. 硅酸盐通报, 2017，36(增): 191-199.

ZHANG Chenyang, WANG Yufei, XANG Zaikui, et al. Research progress in precision machining of silica glass[J]. Bulletin of The Chinese Ceramic Society, 2017 , 36(Suppl.): 191-199.

[5] 苑泽伟, 金洙吉, 李强, 等. CVD 金刚石化学机械抛 光工艺研究 $[J]$. 人工晶体学报, 2016, 45 (1): 73-79. YUAN Zewei, JIN Zhuji, LI Qiang, et al. Study on the chemical mechanical polishing technique of CVD diamond[J]. Journal of Synthetic Crystals，2016， 45 (1): 73-79.

[6] AURICH J C, KIRSCH B, SETTI D, et al. Abrasive processes for micro parts and structures[J]. CIRP Annals, 2019, 68(2): 653-676.

[7] 肖晓兰, 阎秋生, 林华泰, 等. 氮化硅陶瓷球研磨抛光 技术研究进展 $[\mathrm{J}]$. 广东工业大学学报, 2018, 35(6): $18-23$.

XIAO Xiaolan, YAN Qiusheng, LIN Huatai, et al. Research progress of silicon nitride ceramic ball polishing technology [J]. Journal of Guangdong University of
Technology, 2008, 35(6): 18-23.

[8] 袁巨龙, 吴喆, 吕冰海, 等. 非球面超精密抛光技术研 究现状[J]. 机械工程学报, 2012，48(23): 167-177.

YUAN Julong, WU Zhe, LÜ Binghai, et al. Review on Ultra-precision polishing technology of aspheric surface[J]. Journal of Mechanical Engineering, 2012, 48(23): 167-177.

[9] GAVALDA D O, GARCIA L G, LIAO Z, et al. The new challenges of machining ceramic matrix composites (CMCs): Review of surface integrity[J]. International Journal of Machine Tools and Manufacture, 2019, 139: 24-36.

[10] 张志辉, 何丽婷, 孔令豹, 等. 超精密抛光自由曲面光 学的表面生成 $[\mathrm{J}]$. 红外与激光工程, 2010, 39(3): 496-501.

CHEUNG Chifai, HO Laiting, KONG Lingbao, et al. Optical surface generation in ultra-precision polishing of freeform[J]. Infrared and Laser Engineering, 2010, 39(3): 496-501.

[11] 张伟, 李洪玉, 于国戟. 光学元件超精密气囊抛光关键 技术研究现状 [J]. 光学学报, 2009, 29(1): 27-34.

ZHANG Wei, LI Hongyu, YU Guoyu. Current situation of ultra-precision bonnet polishing key technology of optical elements[J]. Acta Optica Sinica, 2009, 29(1): 27-34.

[12] 荣烈润. 超精密研磨抛光方法 [J]. 航空精密制造技术, 2005(2): 4-8.

RONG Lierun. Ultra-precision grinding and polishing method $[\mathrm{J}]$. Aviation Precision Manufacturing Technology, 2005(2): 4-8.

[13] 赵明利, 赵波, 高国富. 超精密研抛及超声波研抛技术 分析[J]. 现代机械, 2006(6): 50-53.

ZHAO Mingli, ZHAO Bo, GAO Guofu. Analysis of ultra-precision polishing and ultrasonic polishing technology [J]. Modern Machinery, 2006(6): 50-53.

[14] 王慧军, 张飞虎, 赵航, 等. 超声波磁流变复合抛光中 几种工艺参数对材料去除率的影响 [J]. 光学精密工程, 2007(10): 1583-1588.

WANG Huijun, ZHANG Feihu, ZHAO Hang, et al. Effects of several technological parameters on material removal in ultrasonic magnetorheological composite polishing [J]. Optics and Precision Engineering, 2007(10): 1583-1588.

[15] 胡锦飞. 磁流变一超声波综合技术抛光氧化锆陶瓷的 研究[D]. 长春: 长春工业大学, 2018.

HU Jinfei. Research on polishing zirconia ceramics with magnetorheological-ultrasonic synthesis technology [D]. Changchun: Changchun University of Technology, 2018. [16] 张占立, 熊明照, 王恒迪, 等. 氮化硅陶瓷滚子磁流变、 
化学与超声复合抛光工艺试验[J]. 轴承, 2016(2): 14-19. ZHANG Zhanli, XIONG Mingzhao, WANG Hengdi, et al. Experiments on magneto-rheological, chemical and ultrasonic composite polishing process of silicon nitride ceramic roller [J]. Bearing, 2016(2): 14-19.

[17] 高航, 吴鸣宇, 付有志, 等. 流体磨料光整加工理论与 技术的发展 [J]. 机械工程学报, 2015, 51(7): 174-187. GAO Hang, WU Mingyu, FU Youzhi, et al. Development of fluid abrasive finishing theory and technology [J]. Journal of Mechanical Engineering, 2015, 51(7): 174-187.

[18] 张雷, 侯汉, 韩艳君, 等. 超声电流变复合抛光试验 $[\mathrm{J}]$. 东北大学学报, 2019, 40(3): 356-359.

ZHANG Lei, HOU Han, HAN Yanjun, et al. Ultrasonic and electrorheological integrated polishing process [J]. Journal of Northeastern University, 2019, 40(3): 356-359.

[19] PETARE A C, JAIN N K. A critical review of past research and advances in abrasive flow finishing process $[\mathrm{J}]$. The International Journal of Advanced Manufacturing Technology, 2018, 97(1-4): 741-782.

[20] KUMAR S S, HIREMATH S S. A Review on abrasive flow machining (AFM) [J]. Procedia Technology, 2016, 25: $1297-1304$.

[21] ZHANG P, DONG Y Z, CHOI H J, et al. Reciprocating magnetorheological polishing method for borosilicate glass surface smoothness[J]. Journal of Industrial and Engineering Chemistry, 2020, 84(25): 243-251.

[22] 陈逢军, 苗想亮, 唐宇, 等. 磨料液体射流抛光技术研 究进展 [J]. 中国机械工程, 2015, 26(22): 3116-3123. CHEN Fengjun, MIAO Xiangliang, TANG Yu, et al. Research progress on abrasive fluid jet polishing technology [J]. China Mechanical Engineering, 2015, 26(22): 3116-3123.

[23] 张朝辉, 耿旭, 李梓万, 等. 化学机械抛光中的接触状 态研究[J]. 表面技术，2020，49(3)：50-56.

ZHANG Chaohui, GENG Xu, LI Ziwan, et al, Contact state study in chemical mechanical polishing[J]. Surface Technology, 2020, 49(3): 50-56.

[24] JONES A R, HULL J B. Ultrasonic flow polishing[J]. Ultrasonics, 1998, 36(1): 97-101.

[25] 万宏强, 韩佩瑛, 葛帅, 等. 工件表面超声振动抛光方 法发展概况[J]. 金刚石与磨料磨具工程, 2018，38(2): 94-100.

WAN Hongqiang, HAN Peiying, GE Shuai, et al. Development of ultrasonic vibration polishing method on workpiece surface $[\mathrm{J}]$. Diamond and Abrasive Engineering, 2008, 38(2): 94-100.

[26] ICHIDA Y, ATO R, MORIMOTO Y, et al. Material removal mechanisms in non-contact ultrasonic abrasive machining[J]. Wear, 2005, 258(1-4): 107-114.
[27] RALCHENKO V G, ASHKINAZI E E, ZAVEDEEV E $\mathrm{V}$, et al. High-rate ultrasonic polishing of polycrystalline diamond films[J]. Diamond and Related Materials, 2016, 66: $171-176$.

[28] 舒晨, 李新和, 卜佳南, 等. 超声磨粒冲击加工去除模 型建立与仿真 $[J]$. 机械科学与技术，2015，34(9): 1360-1364.

SHU Chen, LI Xinhe, BU Jianan, et al. Removal model establishing and simulation of ultrasonic abrasive impacts [J]. Journal of Mechanical Science and Technology, 2015, 34(9): 1360-1364.

[29] YU T, ZHANG T, YANG T, et al. CFD simulation and experimental studies of suspension flow field in ultrasonic polishing[J]. Journal of Materials Processing Technology, 2019, 266: 715-725.

[30] YU T, GUO X, WANG Z, et al. Effects of the ultrasonic vibration field on polishing process of nickel-based alloy Inconel718[J]. Journal of Materials Processing Technology, 2019, 273: 116228.

[31] YU T, ZHANG T, YU X, et al. Study on optimization of ultrasonic-vibration-assisted polishing process parameters $[\mathrm{J}]$. Measurement, 2019, 135: 651-660.

[32] ZHANG T, ZHAO Y, YU T, et al. Study on polishing slurry waste reduction in polishing monocrystalline silicon based on ultrasonic atomization[J]. Journal of Cleaner Production, 2019, 233: 1-12.

[33] 计时鸣, 陈凯, 谭大鹏, 等. 超声空化对软性磨粒流切 削效率和质量的影响 [J]. 农业工程学报, 2017, 33(12): 82-90.

JI Shiming, CHEN Kai, TAN Dapeng, et al. Effect of ultrasonic cavitation on machine efficiency and quality of soft abrasive flow[J]. Transactions of the Chinese Society of Agricultural Engineering, 2017，33(12): 82-90.

[34] 韩帅非, 计时鸣, 陈国达, 等. 耦合超声场的气液固三 相磨粒流多物理场数值模拟研究 [J]. 机电工程, 2017, 34(8): 841-846.

HAN Shuaifei, JI Shiming, CHEN Guoda, et al. Numerical simulation of gas-liquid-solid three-phase abrasive flow with coupled ultrasonic field [J]. Journal of Mechanical and Electrical Engineering, 2017, 34(8): 841-846.

[35] 万宏强, 韩佩瑛, 葛帅, 等. 超声复合振动抛光中材料 去除原理及力学分析[J]. 煤矿机械, 2018, 39(11): 64-67. WAN Hongqiang, HAN Peiying, GE Shuai, et al. Principle of material removal and mechanical analysis in ultrasonic composite vibration polishing [J]. Coal Mine Machinery, 2008, 39(11): 64-67.

[36] 汪帮富, 李华, 殷振, 等. 微磨料汽雾超声抛光加工技 术及试验研究[J]. 制造技术与机床, 2015(1)：33-37. 
WANG Bangfu, LI Hua, YIN Zhen, et al. Technology and experimental study on polishing by micro abrasive ultrasonic mist[J]. Manufacturing Technology \& Machine Tool, 2015(1): 33-37.

[37] 何就, 高萍. 硅片的超声流体喷射抛光试验研究[J]. 机 械设计与制造，2014(9): 129-131.

HE Qing, GAO Ping. Experimental research on ultrasonic fluid jet polishing of silicon wafer[J]. Machinery Design \& Manufacture, 2014(9): 129-131.

[38] BEAUCAMP A, KATSUURA T, TAKATA K. Process mechanism in ultrasonic cavitation assisted fluid jet polishing[J]. CIRP Annals - Manufacturing Technology, 2018, 67: 361-364.

[39] BEAUCAMP A, KATSUURA T, KAWARA Z. A novel ultrasonic cavitation assisted fluid jet polishing system [J]. CIRP Annals, 2017, 66(1): 301-304.

[40] 李道朋, 傅波, 庄文敏. 换能器阵列型超声抛光机理及 声场仿真和试验研究 [J]. 西安交通大学学报, 2020(2): 24-34.

LI Daopeng, FU Bo, ZHUANG Wenmin. Ultrasonic polishing mechanism and sound field simulation and experimental study of transducer array[J]. Journal of Xi'An Jiaotong University, 2020(2): 24-34.

[41] 于兆勤, 郭钟宁, 黄志刚, 等. 流体二维振动加工技术 的试验研究[J]. 中国机械工程, 2008(6): 718-721.

YU Zhaoqin, GUO Zhongning, HUANG Zhigang, et al. Experimental research on the fluid two-dimensional vibration machining technology $[\mathrm{J}]$. China Mechanical Engineering, 2008(6): 718-721.

[42] QI H, CHENG Z, CAI D, et al. Experimental study on the improvement of surface integrity of tungsten steel using acoustic levitation polishing[J]. Journal of Materials Processing Technology, 2018, 259: 361-367.

[43] VENKATESH G, SHARMA A K, KUMAR P. On ultrasonic assisted abrasive flow finishing of bevel gears[J]. International Journal of Machine Tools and Manufacture, 2015, 89: 29-38.

[44] 赵轩达, 祝锡晶, 庞昊斐. 超声辅助磨料流加工的材料 去除机理及参数研究 $[J]$. 热加工工艺, 2017, 46(6): 163-167.

ZHAO Xuanda, ZHU Xijing, PANG Haofei. Research on material removal mechanism and parameters of ultrasound-assisted abrasive flow machining [J]. Thermal Processing Technology, 2017, 46(6): 163-167.

[45] 董志国, 刘建成, 轧钢, 等. 一种圆管内壁超声振动辅 助磨料流精密光整加工方法: 中国专利: CN109333175A[P]. 2019-02-15. DONG Zhiguo, LIU Jiancheng, ZHA Gang, et al. A precision finishing method for abrasive flow assisted by ultrasonic vibration on inner wall of circular tube: Chinese patent CN109333175A[P]. 2019-02-15.

[46] NATARAJAN Y, MURUGESAN P K, MOHAN M, et al. Abrasive waterjet machining process: A state of art of review[J]. Journal of Manufacturing Processes, 2020, 49.

[47] MELENTIEV R, ANG F. Recent advances and challenges of abrasive jet machining[J]. CIRP Journal of Manufacturing Science and Technology, 2018, 22: 1-20.

[48] NGUYEN T, WANG J. A review on the erosion mechanisms in abrasive water jet micromachining of brittle materials $[\mathrm{J}]$. International Journal of Extreme Manufacturing, 2019, 1(1): 012006.

[49] LÜ Z, HUANG C, ZHU H, et al. A 3D simulation of the fluid field at the jet impinging zone in ultrasonic-assisted abrasive waterjet polishing[J]. The International Journal of Advanced Manufacturing Technology, 2016, 87(9-12): 3091-3103.

[50] HOU R, HUANG C, ZHU H. Experimental study on pulsation behavior of the ultrasonic vibration-assisted abrasive waterjet[J]. The International Journal of Advanced Manufacturing Technology, 2017, 91(9-12): 3851-3859.

[51] 王勇. 超声扭转振动辅助磨料水射流抛光陶瓷材料的 工艺研究[D]. 济南: 山东大学, 2015 .

WANG Yong. Study on polishing process of ceramic materials by ultrasonic torsional vibration-assisted abrasive water jet[D]. Jinan: Shandong University, 2015.

[52] SUAUKI H, MORIWAKI $\mathrm{T}$, OKINO $\mathrm{T}$, et al. Development of ultrasonic vibration assisted polishing machine for micro aspheric die and mold[J]. CIRP Annals, 2006, 55(1): 385-388.

[53] SUAUKI H, HAMADA S, OKINO T, et al. Ultraprecision finishing of micro-aspheric surface by ultrasonic two-axis vibration assisted polishing[J]. CIRP Annals, 2010, 59(1): 347-350.

[54] 韩磊. 硬脆材料超声波振动辅助研磨抛光的仿真与试 验研究[D]. 长春: 吉林大学, 2015 .

HAN Lei. Simulation and experimental study on ultrasonic vibration assisted grinding and polishing of hard and brittle materials [D]. Changchun : Jilin University, 2015.

[55] YU T, AN J, YANG X, et al. The study of ultrasonic vibration assisted polishing optical glass lens with ultrasonic atomizing liquid[J]. Journal of Manufacturing Processes, 2018, 34: 389-400.

[56] 赵清亮, 孙智源, 郭兵. 碳化硅圆柱槽微结构表面的化 学机械抛光 [J]. 机械工程学报, 2015, 51(15): 183-189. ZHAO Qingliang, SUN Zhiyuan, GUO Bing. Chemical mechanical polishing of cylindrical silicon carbide groove 
microstructure [J]. Journal of Mechanical Engineering, 2015, 51(15): 183-189.

[57] ZHAO Qingliang, SUN Zhiyuan, GUO Bing. Material removal mechanism in ultrasonic vibration assisted polishing of micro cylindrical surface on $\mathrm{SiC}[\mathrm{J}]$. International Journal of Machine Tools and Manufacture, 2016, 103: 28-39.

[58] TSAI M, YANG W. Combined ultrasonic vibration and chemical mechanical polishing of copper substrates[J]. International Journal of Machine Tools and Manufacture, 2012, 53(1): 69-76.

[59] XU W, CHENG Y, ZHONG M. Effects of process parameters on chemical-mechanical interactions during sapphire polishing[J]. Microelectronic Engineering, 2019, 216: 111029.

[60] XU W, LU X, PAN G, et al. Effects of the ultrasonic flexural vibration on the interaction between the abrasive particles; pad and sapphire substrate during chemical mechanical polishing (CMP)[J]. Applied Surface Science, 2011, 257(7): 2905-2911.

[61] LI L, HE Q, ZHENG M, et al. Improvement in polishing effect of silicon wafer due to low-amplitude megasonic vibration assisting chemical-mechanical polishing[J]. Journal of Materials Processing Technology, 2019, 263: 330-335.

[62] 李亮, 任奕, 李小龙, 等. 兆声辅助化学机械抛光中振 动对抛光液流动的影响 [J]. 科学技术与工程, 2019, 19(3): 79-84.

LI Liang, REN Yi, LI Xiaolong, et al. Effects of vibration on polishing fluid flow in chemical mechanical polishing assisted by sound signals [J]. Science Technology and Engineering, 2019, 19(3): 79-84.

[63] LI L, HE Q, ZHENG M, et al. Contribution of ultrasonic traveling wave to chemical-mechanical polishing[J]. Ultrasonics, 2015, 56: 530-538.

[64] LIU D, YAN R, CHEN T. Material removal model of ultrasonic elliptical vibration-assisted chemical mechanical polishing for hard and brittle materials[J]. The International Journal of Advanced Manufacturing Technology, 2017, 92(1-4): 81-99.

[65] KOBAYASHI N, WU Y, NOMURA M, et al. Precision treatment of silicon wafer edge utilizing ultrasonically assisted polishing technique[J]. Journal of Materials Processing Technology, 2008, 201(1-3): 531-535.

[66] 翟文杰, 翟权. 碳化硅超声-电化学机械抛光试验研究 [J]. 哈尔滨工业大学学报, 2019, 51(1): 16-21.

ZHAI Wenjie, ZHAI Quan. Experimental study on ultrasonic- electrochemical mechanical polishing of silicon carbide[J]. Journal of Harbin Institute of
Technology, 2019, 51(1): 16-21.

[67] 翟文杰, 杨德重, 宫娜. 超声振动条件下碳化硅抛光过 程的分子动力学模拟 $[\mathrm{J}]$. 上海交通大学学报, 2018 , 52(5): 599-603.

ZHAI Wenjie, YANG Dezhong, GONG Na. Molecular dynamics simulation of silicon carbide polishing under ultrasonic vibration [J]. Journal of Shanghai Jiao Tong University, 2008, 52(5): 599-603.

[68] TAN K L, YEO S H. Surface finishing on IN625 additively manufactured surfaces by combined ultrasonic cavitation and abrasion[J]. Additive Manufacturing, 2020, 31: 100938.

[69] TAN K L, YEO S H. Surface modification of additive manufactured components by ultrasonic cavitation abrasive finishing[J]. Wear, 2017, 378-379: 90-95.

[70] ZAREPOUR H, YEO S H. Single abrasive particle impingements as a benchmark to determine material removal modes in micro ultrasonic machining[J]. Wear, 2012, 288: 1-8.

[71] 李小龙. 兆声辅助抛光中抛光液分布特性与抛光机理 研究[D]. 锦州: 辽宁工业大学, 2019.

LI Xiaolong. Study on polishing fluid distribution characteristics and polishing mechanism in sound assisted polishing [D]. Jinzhou : Liaoning University of Technology, 2019.

[72] XU W, LU X, PAN G, et al. Ultrasonic flexural vibration assisted chemical mechanical polishing for sapphire substrate[J]. Applied Surface Science, 2010, 256(12): 3936-3940.

[73] 杨卫平, 吴勇波, 徐家文. 超声椭圆振动-化学机械复 合抛光工具及试验研究 $[J]$. 机械科学与技术, 2008(7): 841-847.

YANG Weiping, WU Yongbo, XU Jiawen. Ultrasonic elliptical vibration-chemical mechanical compound polishing tool and experimental research [J]. Journal of Mechanical Science and Technology, 2008(7): 841-847

[74] 翟文杰, 孙丙镇. 超声辅助抛光过程中流体性能仿真分 析[J]. 哈尔滨工业大学学报, 2017, 49(7): 33-38.

ZHAI Wenjie, SUN Bingzhen. Simulation analysis of fluid performance during ultrasound-assisted polishing [J]. Journal of Harbin Institute of Technology，2017，49(7): 33-38.

[75] 钟敏, 袁任江, 李小兵, 等. 磨粒和抛光垫特性对蓝宝 石超声化学机械抛光的影响 [J]. 中国表面工程, 2018, 31(6): 125-132.

ZHONG Min, YUAN Renjiang, LI Xiaobing, et al. Effect of abrasive and polishing pad characteristics on ultrasonic chemical and mechanical polishing of sapphire [J]. China Surface Engineering, 2008, 31(6): 125-132. 
[76] 翟文杰, 翟权. 超声-化学机械研抛的流体动力学仿真 [J]. 哈尔滨工程大学学报, 2019, 40(12): 2050-2056. ZHAI Wenjie, ZHAI Quan. Fluid dynamics simulation on ultrasonic-chemical mechanical polishing $[\mathrm{J}]$. Journal of Harbin Engineering University, 2019, 40(12): 2050-2056

[77] 吕婷. 基于 CFD 的超声空化对抛光介质运动影响的研 究[D]. 苏州: 苏州大学, 2015 .

LV Ting. Effect of CFD based ultrasonic cavitation on polishing medium movement [D]. Suzhou: Soochow University, 2015.

[78] CURODEAU A, GUAY J, RODRIGUE D, et al. Ultrasonic abrasive $\mu$-machining with thermoplastic tooling[J]. International Journal of Machine Tools and Manufacture, 2008, 48(14): 1553-1561.

[79] FINNIE I. Some reflections on the past and future of erosion[J]. Wear, 1995, 186-187: 1-10.

[80] SLIKKERVEER P J, BOUTEN P C P. Erosion and damage by sharp particles[J]. Wear, 1998, 217(2): 237-250.

[81] MIESZALA M, TORRUBIA P L, AXINTE D A, et al. Erosion mechanisms during abrasive waterjet machining: Model microstructures and single particle experiments[J]. Journal of Materials Processing Technology, 2017, 247: 92-102.

[82] AHMED D H, NASER J, DEAM R T. Particles impact characteristics on cutting surface during the abrasive water jet machining: Numerical study[J]. Journal of Materials Processing Technology, 2016, 232: 116-130.

[83] EITOBGY M, NG E, ELBESTAWI M A. Modelling of abrasive waterjet machining: A new approach[J]. CIRP Annals, 2005, 54(1): 285-288.

[84] WEI H, PENG C, GAO H, et al. On establishment and validation of a new predictive model for material removal in abrasive flow machining[J]. International Journal of Machine Tools and Manufacture, 2019, 138: 66-79.

[85] 朱洪涛. 精密磨料水射流加工硬脆材料冲蚀机理及拋 光技术研究[D]. 济南: 山东大学, 2007.

ZHU Hongtao. Research on erosion mechanism and polishing technology of hard and brittle materials processed by precision abrasive water jet [D]. Jinan: Shandong University, 2007

[86] 冯应恒, 刘润爱, 郭文亮. 超声振动抛光中材料去除原 理与力学分析 $[\mathrm{J}]$. 机械科学与技术, 2020, 39(6): 879-883.

FENG Yingheng, LIU Runai, GUO Wenliang. Principle and mechanical analysis of material removal in ultrasonic vibration polishing $[\mathrm{J}]$. Mechanical Science and Technology[J]. 2020, 39(6): 879-883.

[87] 周忆, 梁德沛. 超声研磨硬脆材料的去除模型研究 [J].
中国机械工程，2005(8): 664-666.

ZHOU Yi, LIANG Depei. Study on the theoretic model of ultrasonic polishing [J]. China Mechanical Engineering, 2005(8): 664-666.

[88] 张成光, 张勇, 张飞虎, 等. 磨料水射流加工去除模型 研究 [J]. 机械工程学报, 2015，51(7): 188-196.

ZHANG Chengguang, ZHANG Yong, ZHANG Feihu, et al. Study on removal model of abrasive water jet machining[J]. Journal of Mechanical Engineering, 2015, 51(7): 188-196.

[89] ZAREPOUR H, YEO S H. Predictive modeling of material removal modes in micro ultrasonic machining $[\mathrm{J}]$. International Journal of Machine Tools and Manufacture, 2012, 62: 13-23

[90] JAFAR R H M, NOURAEI H, EMAMIFAR M, et al. Erosion modeling in abrasive slurry jet micro-machining of brittle materials[J]. Journal of Manufacturing Processes, 2015, 17: 127-140.

[91] CAO Z, CHEUNG C F, REN M. Modelling and characterization of surface generation in fluid jet polishing[J]. Precision Engineering, 2016, 43.

[92] CAO Z, CHEUNG C F. Theoretical modelling and analysis of the material removal characteristics in fluid jet polishing[J]. International Journal of Mechanical Sciences, 2014, 89: 158-166.

[93] 王可, 刘壮, 高长水, 等. 磨料电化学射流加工的材料 去除模型研究 $[\mathrm{J}]$. 机械制造与自动化, 2018，47(3): 127-130.

WANG Ke, LIU Zhuang, GAO Changshui, et al. Study of material removal model of electrochemical abrasive jet machining[J]. Machine Building and Automation, 2008, 47(3): $127-130$

[94] JAMES S, SUNDARAM M. A molecular dynamics study of the effect of impact velocity, particle size and angle of impact of abrasive grain in the vibration assisted nano impact-machining by loose abrasives [J]. Wear, 2013, 303: $510-518$.

[95] 李晓奥, 彭小强, 胡皓. 振动辅助抛光的原理分析与试 验研究[J]. 航空精密制造技术，2016，52(4): 14-19.

LI Xiaoao, PENG Xiaoqiang, HU Hao. Principle analysis and experimental study of vibration assisted polishing $[\mathrm{J}]$. Aerospace Precision Manufacturing Technology, 2016, 52(4): 14-19.

[96] RAJURKAR K P，WANG Z Y，KUPPATTAN A. Micro removal of ceramic material $\left(\mathrm{Al}_{2} \mathrm{O}_{3}\right)$ in the precision ultrasonic machining[J]. Precision Engineering, 1999, 23(2): 73-78.

[97] LIANG Z, WANG X, WU Y, et al. Experimental study on brittle-ductile transition in elliptical ultrasonic assisted 
grinding (EUAG) of monocrystal sapphire using single diamond abrasive grain[J]. International Journal of Machine Tools and Manufacture, 2013, 71: 41-51.

[98] SHARMA A K, VENKATESH G, RAJESHA S, et al. Experimental investigations into ultrasonic-assisted abrasive flow machining (UAAFM) process[J]. The International Journal of Advanced Manufacturing Technology, 2015, 80(1-4): 477-493.

[99] 吕哲. 超声振动辅助磨料水射流抛光冲蚀机理和工艺 技术研究[D]. 济南: 山东大学, 2015 .

LÜ Zhe. Ultrasonic vibration-assisted abrasive water jet polishing erosion mechanism and technology research [D]. Jinan; Shandong University, 2015.

[100] 车翠莲. 硬脆材料曲面的磨料水射流抛光技术研究 [D]. 济南: 山东大学, 2011 .

CHE Cuilian. research on abrasive water jet polishing technology for hard and brittle material surfaces [D]. Jinan; Shandong University, 2011.

[101］鲍慧雪. 蓝宝石光学微腔体的超声水合抛光方法研究 [D]. 长春: 吉林大学, 2016 .

BAO Huixue. study on ultrasonic hydration polishing method for sapphire optical microcavity [D]. Changchun: Jilin University, 2016

[102] 马立. 超声抛光原理研究及超声系统设计 [D]. 苏州; 苏州大学, 2010 .

MA Li. Ultrasonic polishing principle research and ultrasonic system design [D]. Suzhou : Soochow University, 2010.

[103] 赵明. 超声抛光机理研究及其变幅杆设计[D]. 苏州: 苏州大学，2012.

ZHAO Ming. Study on ultrasonic polishing mechanism and luffing rod design [D]. Suzhou : Soochow University, 2012.

[104] QI H, WEN D H. Numerical investigation on particle impact erosion in ultrasonic-assisted abrasive slurry jet micro-machining of glasses[J]. Powder Technology, 2017, 314: 627-634.

[105] QI H, WEN D H. Numerical and experimental study on ultrasonic vibration-assisted micro-channelling of glasses using an abrasive slurry jet[J]. International Journal of Mechanical Sciences，2016，10: 94-107.

[106] 侯荣国. 超声振动辅助磨料水射流脉动行为及其对加 工机理影响机制研究[D]. 济南: 山东大学, 2015.

HOU Rong Guo. Study on pulsation behavior of the ultrasonic vibration assisted abrasive waterjet and its effect of the machining mechanism[D]. Jinan: Shandong University, 2015.

[107] 郑美超. 基于超声振动的硅片边缘抛光研究 [D]. 锦 州: 辽宁工业大学, 2015.

ZHENG Meichao. Research on wafer edge polishing based on ultrasonic vibration[D]. Jinzhou: Liaoning University of Technology, 2015.

[108] 翟科, 任奕, 李亮, 等. 兆声化学机械复合抛光及其 抛光均匀性的实现 $[\mathrm{J}]$. 纳米技术与精密工程, 2017 , 15(6): 538-544.

ZHAI Ke, REN Yi, LI Liang, et al. Realization of polishing uniformity in megasonic vibration assisted chemical mechanical polishing[J]. Nanotechnology and Precision Engineering, 2017, 15(6): 538-544.

作者简介: 李华(通信作者), 男, 1961 年出生, 博士, 教授。主要研究 方向为精密与高效加工技术，超声振动加工技术。

E-mail: lihua@mail.usts.edu.cn 